\title{
Förderung von Gewerbe und Industrie in der schweizerischen Entwicklungszusammenarbeit
}

La promotion artisanale et industrielle dans la coopération suisse au développement

Hilmar Stetter and Jean-Pierre Wolf

\section{OpenEdition}

\section{Journals}

Electronic version

URL: http://journals.openedition.org/sjep/1074

DOI: $10.4000 /$ sjep. 1074

ISSN: 1663-9677

Publisher

Institut de hautes études internationales et du développement

Printed version

Date of publication: 1 janvier 1986

Number of pages: 191-225

ISSN: 1660-5926

\section{Electronic reference}

Hilmar Stetter und Jean-Pierre Wolf, «Förderung von Gewerbe und Industrie in der schweizerischen Entwicklungszusammenarbeit », Schweizerisches Jahrbuch für Entwicklungspolitik [Online], 6 | 1986, Online erschienen am: 09 März 2013, abgerufen am 08 September 2020. URL : http:// journals.openedition.org/sjep/1074; DOI : https://doi.org/10.4000/sjep.1074 


\title{
FOERDERUNG VON GEWERBE UND INDUSTRIE IN DER SCHWEIZERISCHEN ENTWICKLUNGSZUSAMMENARBEIT
}

\author{
Hilmar Stetter / Jean-Pierre Wolf
}

Résumé: La promotion artisanale et industrielle dans la coopération suisse au développement

En politique de développement, la discussion sur les stratégies d'industrialisation compte parmi les thèmes les plus anciens et les activités dans le secteur artisanal/industriel font partie du répertoire classique de l'aide au développement. Toutefois, ce n'est que vers les années 1980 qu'un regain d'intérêt de la part des organismes de développement se manifeste et que leurs efforts porteront prioritairement sur ce secteur. II va sans dire que la situation économique difficile de la plupart des pays en développement, la croissance de leur endettement et l'augmentation du chômage sont des raisons suffisantes pour favoriser et encourager le secteur clef qu'est la promotion artisanale et industrielle.

L'objectif de cet article est de présenter, d'une part, les activités typiques menées par les organisations suisses à la promotion de l'artisanat et de l'industrie dans le cadre de la coopération au développement et, d'autre part, de montrer au lecteur les dimensions de l'enjeu de cette promotion. 
Dans le chapitre introductif, la promotion artisanale/industrielle est placée dans son contexte historique. Suit un inventaire des différentes notions utilisées pour désigner les sous-secteurs qui font l'objet de ces interventions promotionnelles.

Le deuxième chapitre présente les activités dans le secteur artisanal/industriel de cinq agents de coopération au développement. I/ s'agit de la DDA, Swisscontact, Swissaid, FUNDES et le bureau de I'ONUDI à Zurich. Cette présentation est suivie d'une comparaison montrant les différences et ressemblances des activités menées par les organismes sélectionnés.

Les questions méritant une réflexion approfondie sur les résultats escomptés de la promotion du secteur artisanal/industriel font l'objet du troisième chapitre. II s'agit de questions relatives au type de relations entre les sous-secteurs de l'économie; aux différentes conceptions de la gestion d'entreprise; à la participation des bénéficiaires, aux choix des technologies et aux influences conjoncturelles sur les résultats de la promotion. 
Die Förderung von Handwerk, Gewerbe und Kleinindustrie, von uns GIF (Gewerbe/Industrie-Förderung) genannt, gehört zu den klassischen Sektoren der Entwicklungszusammenarbeit und die Strategien der Industrialisierung sind klassische Themen der Entwicklungspolitik. Während die Notwendigkeit der Massnahmen in diesem Sektor unbestritten ist, zeichnen sich in der Art der Förderung und in ihren Zielen unter den schweizerischen Entwicklungsorganisationen Verschiedenheiten in der Konzeption, Durchführung und in den gemachten Erfahrungen ab.

Gerade im Hinblick auf ein derzeit wachsendes Interesse internationaler und schweizerischer Entwicklungsorganisationen und der Entwicklungspolitik an diesem Sektor kann die Darstellung und Diskussion bisheriger Erfahrungen eine Orientierung bieten und eine Informationslücke schliessen.

\section{EINLEITUNG}

Ueber Massnahmen zur Gewerbe- und Industrieförderung (GIF) im Rahmen der Entwicklungszusammenarbeit (EZA) versuchen immer mehr Entwicklungsorganisationen und -agenturen Entwicklungsziele wie folgende zu erreichen:

- Erhaltung bestehender und Schaffung neuer Beschäftigungsmöglichkeiten.

- Schaffung und Umverteilung von Einkommen.

- Lokale Wertschöpfung durch Verwendung und Verarbeitung lokaler (besonders landwirtschaftlicher) Rohmaterialien.

- Importsubstitution.

- Dezentralisierung und Regionalentwicklung (als Mittel gegen die rasch fortschreitende Urbanisierung).

- Verbesserung der Ausfuhrmöglichkeiten von Gütern und Dienstleistungen.

Kredite, Ausbildung, Unternehmensberatung, Aufbau von Infrastrukturen, gemeinsame Rohstoffeinkäufe und Vermarktungsanstrengungen - dies sind einige der wichtigeren Instrumente, die bei GIF-Massnahmen im Vordergrund stehen. Gesicherte Erfahrungen über den Erfolg der eingesetzten Instrumente sind besonders in den am wenigsten entwickelten Ländern nicht häufig und oft widersprüchlich. Das hat nicht zuletzt mit der Heterogenität dessen zu tun, was zusammengefasst unter der Bezeichnung "gewerblicher und kleinindustrieller Sektor» Gegenstand der Entwicklungsanstrengungen ist.

In diesem Beitrag wird in einem ersten Teil einführend der geschichtliche Kontext erläutert, durch den die Aktualität und Ausrichtung der GIF in den 80er Jahren verständlich wird: der industrielle Aufbau innerhalb der Modernisierungsstrategie der 60er Jahre; die Hinwendung zu kleineren Industrieprojekten in der ländlichen Entwicklung der 70er Jahre. Dann ist es 
unumgänglich, sich mit begrifflichen Fragen kurz zu beschäftigen: Welchen Raum decken GIF-Massnahmen ab? Es wird festgestellt, dass es keine einheitlichen Definitionen für die Vielzahl der geförderten Bereiche und Erwerbsformen gibt; die Abgrenzungen sind fliessend und werden vom Förderungsinteresse bestimmt. Schliesslich soll der Leser einen Ueberblick über die Interventionsebenen und Förderungsansätze erhalten, die bei der GIF im allgemeinen eine Rolle spielen: die Förderung von Einzelunternehmen und von ganzen Untersektoren und die Verbesserung von institutionellen Rahmenbedingungen; dabei kommen unterschiedliche Ansätze zur Anwendung, sehr punktuelle bis mehr oder weniger integrierte.

Nach diesem allgemeinen Teil werden im zweiten Teil Interventionen zur GIF, die von der Schweiz ausgeführt werden, beschrieben und miteinander verglichen: jene der DEH, von Swissaid, Swisscontact, FUNDES und des UNIDO-Büros in Zürich. Mit der Beschreibung der fünf Organisationen ist die von der Schweiz aus geleistete GIF nicht vollständig dargestellt. Die von den erwähnten Organisationen ausgehenden GIF-Massnahmen können jedoch als typisch für Aktivitäten in diesem Bereich betrachtet werden und decken in diesem Sinne das Spektrum schweizerischer Anstrengungen innerhalb der EZA ab (1). Durch die Darstellung der Profile und Beziehungen der einzelnen Organisationen in zwei Schemas werden Unterschiede und Gemeinsamkeiten im Bereich der GIF sichtbar gemacht.

Schliesslich werden in einem dritten Teil Fragen aufgeworfen zu Aspekten, die u.E. weiterer Reflexion bedürfen: Die Beziehungen zwischen dem durch die GIF geförderten und dem nicht geförderten Untersektor der Wirtschaft; die Ergänzung betriebswirtschaftlicher Konzepte; die Wahl und der Transfer der benötigten Technik; die Beachtung sozialer Fragen innerhalb des geförderten Untersektors; die Mitwirkung der Betroffenen; und der Einfluss von Konjunktur und Marktverhältnissen auf den Erfolg von GIFMassnahmen.

\section{KONZEPTUELLER RAHMEN}

\subsection{Entwicklungsetappen}

GIF-Massnahmen sind seit Beginn der EZA ein Thema der Diskussion über Entwicklung und Entwicklungsstrategien. Die Akzente haben sich dabei allerdings im Laufe der Jahre verlagert aufgrund veränderter wirtschaftlicher und sozialer Rahmenbedingungen und Aenderungen in der Thematisierung gewisser Entwicklungsaspekte innerhalb der Entwicklungsdiskussion.

\section{Anmerkung :}

1. Nicht dargestellt sind die Organisationen: Helvetas, die in Nepal über eine lange Erfahrung im Aufbau eines kleinindustriellen Pionierbetriebs verfügt; das SAH, das HEKS, das Fastenopfer und kleinere kirchliche Organisationen, welche keine 
eigenständigen GIF-Programme unterhalten, aber zum Teil zahlreiche kleinere GIF-Projekte in Bereichen unterstützen, die für sie prioritär sind; Intercooperation, die sich neben ihren Schwerpunkten Landwirtschaft und Forstwirtschaft zunehmend für die GIF interessiert. Auch das BAWI ist hier nicht weiter berücksichtigt worden, obschon es im Rahmen des Kredites für handels- und wirtschaftspolitische Massnahmen und in Zusammenarbeit mit der DEH GIF-Massnahmen mitgestaltet und mitträgt. Allerdings haben wir ein vom BAWI finanziertes Förderungsinstrument, das Zürcher UNIDO-Büro, in unseren Artikel einbezogen, um das Spektrum der Massnahmen besser zeigen zu können.

Nehmen wir zur Illustration die handwerkliche Herstellung und Bearbeitung von Gütern, die Tradition hat und in der Regel noch verbreitet ist, wie zum Beispiel das westafrikanische Textilhandwerk mit seinen hoch entwikkelten Webe- und Färbtechniken und seiner gesellschaftlichen Bedeutung als Kaste. Das traditionelle Handwerk ist von verschiedenen Entwicklungen her in Bedrängnis geraten. Einmal hat der schnelle gesellschaftliche Wandel in den Entwicklungsländern starke Veränderungen in den Bedürfnissen mit sich gebracht, z.B. durch die Urbanisierung innerhalb des Transportwesens oder durch die ländliche Mechanisierung auf dem Gebiet der Herstellung und des Unterhalts von neuen Geräten und Maschinen. Dadurch entstanden einerseits völlig neue Kleingewerbe, wie die Ersatzeilhändler und Reparateure von Fahrrädern und Motorfahrrädern. Andererseits wurden an die herkömmlichen Handwerksbetriebe weitgehende Anpassungsforderungen gestellt, etwa die Einführung von Schweisstechniken in ländlichen Schmiedewerkstätten. Gleichzeitig haben sich aber die Produktionsbedingungen durch die starke Importkonkurrenz bei sinkender Kaufkraft in der Bevölkerung verschlechtert. Als Folge solcher Veränderungen ist das Handwerk in eine prekäre Lage geraten, gekennzeichnet durch einen sinkenden Status und eine schwindende Autonomie.

Unter andern gesamtwirtschaftlichen Bedingungen hätte man in diesem Prozess der Marginalisierung noch eine notwendige Strukturbereinigung sehen können, wie er von den Modernisierungsstrategen begrüsst worden ist. Im Kontext einer allgemeinen Verarmung und Massenarbeitslosigkeit wird diese Situation entwicklungspolitisch als negativ beurteilt und mit gezielten Förderungsmassnahmen zu korrigieren versucht.

Vereinfachend teilen wir nachfolgend die Entwicklung, welche die GIF in den letzten 30 Jahren gemacht hat, in drei Etappen ein, die ungefähr mit den drei Entwicklungsdekaden seit Ende der 50er Jahre übereinstimmen.

- Die im Aussenhandel mit den Industrieländern sich ständig verschlechternden "terms of trade» bilden den ersten starken Anstoss für die Industrialisierung der Entwicklungsländer. Entwicklungspolitiker und Aufbauplaner orientieren sich dabei an den Standards von Unternehmen in Industriestaaten, d.h. an grösseren und modernen Einheiten. Das «traditionelle» Handwerk und bereits bestehende Kleinwerkstätten werden von den Planern kaum zur Kenntnis genommen. Sie gehen davon aus, dass der informelle und kleinindustrielle Sektor durch den Aufbau eines modernen Sektors schrumpfen und schliesslich verschwinden werde, sofern er nicht kom- 
plementär und untergeordnet zu den neuen Betrieben gewisse Leistungen zu erbringen vermag. Weitere Merkmale der industriellen Modernisierungsstrategie sind die Promotionsrolle, die dem Staat zugedacht wird, der hohe Kapitalbedarf, der mit ihr notwendigerweise verbunden ist, und eine gewisse Vernachlässigung des Beschäftigungsaspekts. Man nimmt an, dass sich die Frage der Unterbeschäftigung automatisch mit der Industrialisierung lösen werde, ähnlich wie während des Industrialisierungsprozesses der traditionellen Industrienationen.

- Die Modernisierungsstrategie hat die in sie gesetzten Erwartungen weitgehend nicht zu erfüllen vermocht. Vor dem Hintergrund einer wachsenden Massenarmut und grossräumiger Hungerkatastrophen, wie im Sahel anfangs der 70er Jahre, werden in der zweiten Entwicklungsdekade die Strategien zur Befriedigung menschlicher Grundbedürfnisse formuliert. Landwirtschaft und ländliche Entwicklung rücken dabei ins Zentrum der Förderungsmassnahmen; einerseits wegen der Wichtigkeit, die der Ernährung der schnell wachsenden Bevölkerung zukommt, andererseits, weil ein bedeutender Anteil der Beschäftigten in der Landwirtschaft ihr Auskommen findet und dort immer noch ein zu geringen Kosten erschliessbares Beschäftigungspotential liegt. Der Unterstützung von Handwerksförderungs- und Kleinindustrieprojekten kommt insofern Bedeutung zu, als sie der landwirtschaftlichen Produktion vor- oder nachgelagert sind; also z.B. die Modernisierung von Schmiedewerkstätten als Beitrag zur Herstellung und Reparatur der Geräte für die ländliche Mechanisierung oder von Müllereien, Gerbereien und Käsereien zur lokalen Verarbeitung landwirtschaftlicher Erzeugnisse.

Die Verschuldungskrise, das Ausmass der offenen und versteckten Arbeitslosigkeit und die ungebremste Abwanderung in die Städte geben den GIF-Massnahmen ein neues Gewicht. In der Verarmung breiter Bevölkerungsschichten liegt politischer und sozialer Konfliktstoff. Die Entwicklungsstrategien der 80er Jahre müssen aufzeigen, wie in grossem Umfang vor allem für die städtischen Massen Einkommensmöglichkeiten geschaffen werden können. Nach den ernüchternden Erfahrungen mit dem Aufbau eines kohärenten modernen Industriesektors richtet sich die Aufmerksamkeit heute notwendigerweise auf die zahlreichen und vielfältigen Betätigungen, mit denen grosse Teile der nicht landwirtschaftlichen und nicht im modernen Sektor beschäftigten Bevölkerung schlecht und recht ihren Lebensunterhalt bestreiten müssen. Die geringe Bedeutung der Leistungen des Einzelnen, die vielen Fluktuationen und die Vielfalt der anzutreffenden Situationen haben es der Wissenschaft, vorab der Statistik, schwer gemacht, die Bedeutung des quantitativ wichtigen, «informellen» und «nicht strukturierten» Sektors überhaupt zu sehen und zu erfassen. Dazu kommt, dass viele dieser Erwerbsformen nicht «regelkonform» sind, wenn sie z.B. an den Anforderungen der Gewerbe- und Handelsgesetzgebung oder der Steuer- und Sozialgesetzgebung gemessen werden; diese Marginalität schliesst die Kleinst- und Kleinunternehmer ihrerseits vom Zugang zu öffentlichen Institutionen aus, allen voran vom Kredit- und Ausbildungswe- 
sen. Mit einer gezielten Förderung soll die Produktivität und Qualität der erwähnten Betätigungen verbessert und stabilisiert und dadurch die Beschäftigungslage und das Einkommen gesichert und verbessert werden. Daneben werden weitere Zielsetzungen, wie die eingangs aufgeführten, verfolgt.

\subsection{Zu den Begriffen}

Die erste Schwierigkeit besteht in der Einführung einer adäquaten Bezeichnung für das Ganze. Der Begriff der GIF wird von uns als Oberbegriff für eine Reihe von sehr unterschiedlichen Massnahmen gebraucht, die innerhalb der EZA im ausgedehnten Bereich des Gewerbes und der Industrie vorgenommen werden und Gegenstand dieses Artikels sind. Die zweite Schwierigkeit besteht darin, dass ohne zusätzliche Präzisierungen dessen, worauf sich diese Massnahmen beziehen, die Verwendung des Begriffs nicht sehr aussagekräftig ist; leider besteht aber auch über die Untergliederung und die Einheiten, die den gewerblichen und industriellen Sektor ausmachen, kein Konsens. Ausgehend vom vielfältigen Sprachgebrauch versuchen wir unten, einige Gliederungsmöglichkeiten aufzuzeigen. Dabei taucht eine dritte Schwierigkeit auf, weil unsere Begriffe nicht immer zur Realität passen, die sie erfassen möchten: Wie bezeichnen wir z.B. eine Erwerbsform, die aus den unterschiedlichsten Aktivitäten sowohl im primären, wie im sekundären und tertiären Sektor besteht? Andere Begriffe, wie etwa der des «nicht strukturierten Sektors», erweisen sich bei näherem Hinsehen als ethnozentrisch vorbelastet und können falsche Vorstellungen wecken.

Dichotomische Konzepte, z.B. jenes des «traditionellen» und des «modernen» Untersektors, sind als erste Umschreibung und Annäherung an eine Zielgruppe und ihre Problematik verbreitet. Daneben entstehen Begriffe etwa aufgrund eines einzelnen messbaren Kriteriums, wie z.B. die Unterscheidung der Betriebe nach ihrem Umsatz.

- Der informelle Untersektor: Dem Begriff ist das Bild einer dualistischen Wirtschaft zugrunde gelegt mit einem dominierenden, die Entwicklung bestimmenden, und einem abhängigen Untersektor. Der informelle Untersektor umschliesst alle auf Einkommen abzielenden wirtschaftlichen Betätigungen, die sich nicht an die bestehende Gesetzgebung und die administrativen Vorschriften halten und (noch immer) kaum von der Statistik erfasst sind. Der Sektor umfasst hauptsächlich sich selber beschäftigende, auf die Bestreitung des Lebensunterhalts orientierte Produzenten von Gütern und Dienstleistungen, gemeinwirtschaftlich tätige Vereinigungen, die ausserhalb oder parallel neben dem Markt tätig sind, die Hauswirtschaft, die Schwarzarbeit.

Kernproblem für den so umschriebenen Sektor ist der Zugang zu den öffentlichen Institutionen, der dem Sektor wegen seiner Marginalität verwehrt ist, und die Gestaltung des normativen Rahmens für wirtschaftliche Betätigungen allgemein. Die Haltung der Behörden gegenüber dem infor- 
mellen Sektor ist teilweise noch immer von Unkenntnis und Repression gekennzeichnet.

- Der nicht strukturierte Untersektor: Der Begriff will im wesentlichen dieselbe Realität beschreiben, die auch dem Konzept des «informellen Untersektors» zugrunde liegt. Der Akzent wird auf den Aspekt der Organisation gelegt : diese wäre - gemäss der Auffassung der Autoren des Begriffs - nicht vorhanden im Vergleich mit dem "strukturierten» Untersektor der Wirtschaft. Die Kernfrage dreht sich hier um die Weckung von Synergien, die oft über die Förderung der Selbstorganisation und der Selbsthilfe unter den Betroffenen erreicht werden soll, mit dem Ziel, gemeinsame Investitionen vorzunehmen, die Marktorientierung zu verbessern, das Produktionsvolumen zu erhöhen, die Arbeitsproduktivität zu steigern, u.a.m.

- Der traditionelle und der moderne Untersektor: Hier beziehen sich die Unterscheidungsmerkmale auf die Technologiestufe, die Arbeitsintensität, die Art der Betriebsführung, das Verhältnis unter den Mitarbeitern, das Verhältnis zur Kundschaft, das Selbstverständnis des Meisters oder Unternehmers bezüglich seiner gesellschaftlichen und wirtschaftlichen Rolle und die gesellschaftlichen Erwartungen an ihn. Zur Illustration denke man an die Bierherstellung, die traditionellerweise etwa als Hirsebier von Frauen kontrolliert wurde, heute aber von einigen industriellen Brauereien immer mehr übernommen wird.

Es ist allerdings nicht so, dass sich die GIF immer an den informellen/ nicht strukturierten oder traditionellen Untersektor richten würde. Gerade ältere Projekte und solche mit staatlichen Partnern wenden sich durchaus an Werkstätten und Unternehmen im formellen und modernen* Untersektor.

Schema 1: Dichotomische Konzepte

\begin{tabular}{|c|lc|}
\hline nicht strukturierter & in & - strukturierter \\
- informeller & 1 & - formeller \\
- traditioneller & 1 & - moderner \\
UNTERSERTOR & 1 & UNTERSERTOR \\
\hline
\end{tabular}

- Kleinst-, Klein- und mittlere Unternehmen: Im angelsächsischen Sprachraum werden alle auf die Erzielung von Einkommen ausgerichteten Betätigungen im Zusammenhang mit der GIF einheitlich als "Unternehmen» bezeichnet, ungeachtet ihrer Grösse, Dauerhaftigkeit und Komplexität. Das ist eine bequeme Sprachregelung. Die GIF richtet sich an die kleinsten, kleinen und mittleren Unternehmen: "micro- and small scale enterprises" (SSE) oder "small and medium scale enterprises» (SME). Das häufigste und 
einfachste Klassierungsmerkmal ist die Anzahl von Mitarbeitern im Unternehmen, das aber von Projekt zu Projekt sehr unterschiedlich gehandhabt wird: je nach Kontext darf z.B. ein Kleinunternehmen höchstens 5 oder höchstens 50 Mitarbeiter aufweisen. Der Satz von betrieblichen Unterscheidungsmerkmalen kann je nach der Stossrichtung der GIF beliebig verändert werden: Arbeits- und Kapitalintensität, interne Arbeitsteilung, Arbeitsproduktivität, Betriebsjahre, Betriebsmanagement, Besitzverhältnisse, Marktkenntnisse und Marktorientierung, Produktionsvolumen, Umsatz, Verhältnis zu vor- und nachgelagerten Betrieben, u.a.m. Die einzelnen GIF-Massnahmen ergeben sich dabei aus einer Analyse der Stärken und Schwächen der Unternehmen aufgrund des ausgewählten Kriterienrasters, also z.B. Steigerung der Arbeitsproduktivität, Verbesserung des Managements, Erleichterung des Marktzugangs, Ausschaltung saisonaler Schwankungen im Geschäftsvolumen.

Schema 2: Verteilung nach Unternehmensgrössen

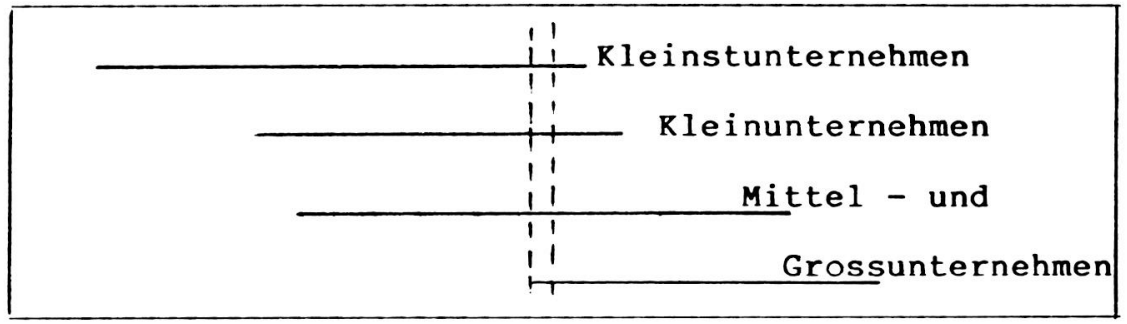

- Der rurale und der urbane Untersektor: Aufgrund der Unterschiede zwischen den beiden Untersektoren kann es sinnvoll sein, dass bei einer entsprechenden Zielsetzung sich die GIF nur an einen von ihnen richtet. Solche Unterschiede sind vorwiegend: die starke Verbindung zur Landwirtschaft und der hohe Anteil von Frauen im ländlichen Untersektor, seine Komplementarität zur landwirtschaftlichen Produktion und die Bedeutung saisonbezogener Aktivitäten.

Schema 3: Räumliche Verteilung

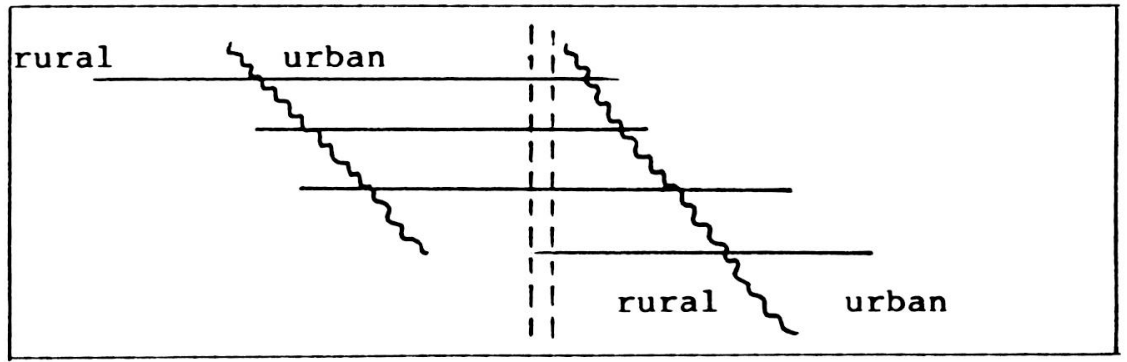


Schliesslich ist zu vermerken, dass sich die GIF nicht ausschliesslich auf den sekundären Wirtschaftssektor, d.h. die handwerklich/industrielle Bearbeitung von Rohmaterialien und Zwischenprodukten, beschränken muss. Je nach Zielsetzung werden auch Teilbereiche des primären Sektors, etwa Fischer- oder Minenarbeitergenossenschaften, oder des Dienstleistungssektors, z.B. das städtische, private Transportwesen, eingeschlossen. Die klassische Aufteilung der Wirtschaft in die drei Sektoren ist insofern unpraktisch, als gerade im informellen/nicht strukturierten und im ländlichen Bereich polyvalente Erwerbsformen häufig sind; man denke an den Bauern/Händler/Handwerker oder an den Handwerker/Kleinhändler/Geldleiher.

Durch die Einführung einiger wesentlicher Begriffe soll dem Leser die Weite des von der GIF betroffenen Feldes gezeigt werden.

Es ist uns klar, dass im konzeptuellen Bereich weitere Klärungen und Entwicklungen notwendig sind.

\subsection{Interventionsebenen und -ansätze}

Wir unterscheiden drei Interventionsebenen: Auf der untersten werden einzelne Unternehmen gefördert; das geschieht weniger selten, als man annehmen könnte. Die Interventionen auf der zweiten Ebene richten sich an den Sektor als solchen, und auf der dritten Ebene geht es um die Schaffung günstiger institutioneller Rahmenbedingungen als Beitrag zur GIF.

Wir beobachten im wesentlichen vier Ansätze, die vom punktuellen bis zum integrierten Vorgehen reichen.

\section{Interventionsebenen}

- Die direkte Unterstützung einzelner Unternehmen: Massnahmen wie Aus- und Weiterbildungsleistungen, Hilfe in der Produktvermarktung, Oeffnung des Zugangs zu Krediten richten sich direkt an einzelne Unternehmen, die bestimmte Kriterien erfüllen. Mit vielen dieser Massnahmen wird die Verbesserung des technologischen Niveaus angestrebt; andere zielen auf die Verbesserung der unternehmerischen Fähigkeiten ab. In die Kategorie der direkten Unterstützungen fällt auch der Aufbau vollkommen neuer Betriebe, die die Rolle von Pionierunternehmen in ausgewählten Regionen oder Branchen übernehmen sollen.

Die Strategie charakterisiert sich dadurch, dass zu den Hauptaufgaben der GIF-Programme die Identifikation, Beurteilung, Selektion, Betreuung und Begleitung der Zielbetriebe gehört, z.B. jener, die als kreditwürdig gelten sollen. $\mathrm{Da}$ in der Regel nicht alle Unternehmen eines Untersektors den Kriterien genügen können, wird in Kauf genommen, dass sich die Konkurrenz zwischen den geförderten und den nicht geförderten Einheiten erhöht. Das kann zur weiteren Marginalisierung oder gar zur Elimination der schwächsten Unternehmen führen. 
- Die Förderung von ganzen Untersektoren: Massnahmen auf dieser Ebene richten sich an die Gesamtheit der Unternehmen bestimmter Branchen und/oder Regionen. Im Mittelpunkt solcher Massnahmen stehen Organisationsprozesse und die Identifikation und Unterstützung von gemeinsamen Anliegen, z.B. Zusammenschlüsse in Genossenschaften, Berufsverbänden und Gewerbekammern; die Durchführung von Ausstellungen und Messen oder die gemeinsame Anschaffung von Geräten und Maschinen und die Führung kollektiver Werkstätten, der organisierte Ankauf von Rohmaterialien und Ersatzteilen und die gemeinsame Vermarktung individuell hergestellter Produkte. Der sich am gesamten Untersektor orientierende Approach umfasst häufig die Förderung von Selbsthilfemassnahmen. Im Vordergrund stehen dabei der Aufbau und die Durchführung von Ausbildungsprogrammen und von Institutionen zur Selbstfinanzierung.

- Die Verbesserung der institutionellen Rahmenbedingungen: Im informellen/nicht strukturierten Bereich der Volkswirtschaft gehören Verbesserungen auf dieser Ebene oft zu den wichtigsten Massnahmen oder zumindest Vorbedingungen für das Gedeihen von Kleinst- und Kleinunternehmen. Sie müssen selbstverständlich von den politischen und administrativen Körperschaften des Entwicklungslandes selbst durchgeführt werden. Die EZA kann dabei nur eine untergeordnete Rolle spielen. Allerdings machen heute immer häufiger ausländische Donatoren die Finanzierung von GIF-Massnahmen von Fortschritten bei der Verbesserung von institutionellen Rahmenbedingungen abhängig.

Ausgangspunkt für die Forderung nach Fortschritten auf dieser Ebene ist die Feststellung, dass Kleinst- und Kleinunternehmer ihre Wirtschaftsaktivität aufrechthalten und anpassen können, obschon sie ganz erheblich durch den institutionellen Rahmen benachteiligt werden. Verbesserungen in den Bereichen der Zinspolitik, der Investitionspolitik, der Berufsbildungspolitik, bei der Erstellung von Infrastrukturen, bei den Vorschriften für Registraturen und Zulassungen, usw., können für solche Unternehmen an sich einen erheblichen Entwicklungseffekt nach sich ziehen.

Veränderungen in den institutionellen Rahmenbedingungen setzen auf allen Ebenen des politisch-administrativen Apparats eine positive Wahrnehmung der kleinen Wirtschaftseinheiten im informellen/nicht strukturierten Bereich und die Kenntnis ihrer volkswirtschaftlichen Bedeutung und ihres Entwicklungspotentials voraus. Eine solche Wahrnehmung ist ihrerseits eine Bedingung für das günstige politische Klima, durch welches Veränderungen im institutionellen Rahmen möglich werden.

\section{Ansätze}

- Bei den punktuellen Ansätzen geht es um die schwergewichtige Förderung isolierter Aspekte, die als Hebel für Veränderungen in umliegenden Bereichen betrachtet werden. Das wichtigste Beispiel ist die Berufsbildung: ein alter und wegen der Ueberschaubarkeit des Gegenstandes beliebter Förderungsansatz. Die Berufsbildung beschränkt sich bisher vorwiegend 
auf die formelle Ausbildung und bereitet den Lehrling auf ein Berufsleben im modernen Untersektor vor. Trotz der Bedeutung und Verbreitung von Berufsbildungsmassnahmen werden wir in diesem Artikel nicht speziell darauf eingehen.

- Bei den kombinierten Ansätzen geht es um mehrere Massnahmen, die in einem Förderungspaket zusammengefasst sind. Solche Pakete entstehen oft aus punktuellen Ansätzen heraus, die in sich immer die Möglichkeit der Ausweitung bergen, z.B. indem einem Berufsbildungssprojekt eine Ausrüstungskomponente angehängt wird, über welche promovierten Berufsleuten das notwendige Werkzeug zugänglich gemacht wird; daraus kann seinerseits das Bedürfnis nach einer Kreditkomponente entstehen, usw.

- Ansätze, die eine vertikale Integration anvisieren, zeichnen sich durch eine Förderungslinie aus, die in eine ganze Produktionskette gezogen wird. Diese kann von der Rohstoffgewinnung bis zur Fertigung des Enderzeugnises gehen, z.B. von der Holzgewinnung über die Sägereien bis zu den Schreinereien.

Solche komplexen Massnahmen werden in Situationen durchgeführt, wo es um den Aufbau einer neuen Produktionslinie geht.

- Schliesslich wird die GIF auch als Bestandteil von regionalen Entwicklungsprogrammen eingesetzt; z.B. in integrierten ländlichen Projekten, wo neben der landwirtschaflichen Produktion, den Infrastrukturbauten, der Gesundheitsvorsorge u.a.m., auch das Handwerk und lokale Kleinunternehmen gefördert werden. Solche Projekte sind seit Mitte der 70er Jahre häufig.

\section{DIE AKTIVITAETEN SCHWEIZERISCHER ENTWICKLUNGS- ORGANISATIONEN}

\subsection{Einige bisherige Erfahrungen}

Ohne zu den entwicklungspolitischen Prioritäten der ersten Stunden zu gehören, haben im Rahmen der von der Schweiz aus geleisteten EZA Projekte zur GIF schon früh Unterstützung gefunden. Bereits in den frühen 60er Jahren wurden wichtige und langfristige Aktivitäten angefangen.

Erinnert sei an die breiten Käsereiprogramme des damaligen DftZ in Asien und Lateinamerika; an das BYS in Nepal, wo SATA (Helvetas) mit einem staatlichen Partner ein Kleinindustrie-Projekt aufbaute, von dem Teile auch noch heute unterstützt werden; an die TRAFIPRO in Rwanda, die als genossenschaftlicher Grossverteiler von Konsumgütern von der DftZ/DEH auf verschiedene Weise während vielen Jahren gefördert wurde. Daneben entstanden etliche Handwerkerförderungsprojekte quasi als Nebenprodukt zu Projekten, zu deren Realisierung nicht die entsprechenden Fachleute zur Verfügung standen, z.B. bei Bauprojekten, welche Ausbildungsprojekte für Baufachleute - Maurer, Zimmerleute, Spengler - nach sich zogen. 
Besonders in den am wenigsten entwickelten Ländern, wo die GIF oft von weit unten beginnen muss, war die Durchführung entsprechender Projekte meistens schwierig. Aus der Erfahrung der Frühzeit lassen sich diese Schwierigkeiten folgendermassen resümieren :

- Die Langfristigkeit der Massnahmen: Etliche dieser Aktionen müssen auch heute noch unterstützt werden; eine frühzeitige Uebergabe ist mit hohem Risiko von Zusammenbrüchen der aufgebauten Werkstätten und Unternehmen verbunden. Bei Projektbeginn war man sich oft über die zeitliche Dimension der Verpflichtung nicht bewusst.

- Die Trägerschaft : die Partner von frühen GIF-Projekten waren halbstaatliche oder staatliche Organismen, wodurch unternehmerische Entscheidkriterien es immer schwer hatten, sich gegen rein administrative Kriterien durchzusetzen. Daneben war auch die Identifikation von guten Unternehmerpersönlichkeiten und deren langfristige Einbindung in die Projekte schwierig.

- Die konzeptuellen und organisatorischen Voraussetzungen bei den Entwicklungsorganisationen: Sie waren alles andere als gut. Pragmatisches Verhalten entsprach am besten einem eher zufällig entstandenen und langsam wachsenden GIF-Programm. An den Zentralen genügten die Instrumente und die fachlichen Qualifikationen der Mitarbeiter, die dazu noch häufig wechselten, kaum für eine konsequente Erfahrungsauswertung. Im Felde sind die Experteneinsätze zu kurz - gemessen an der Langfristigkeit und Komplexität der Aufbauarbeit - und Spezialisten müssen viel Zeit für Aufgaben aufwenden, die sich ausserhalb ihres Fachbereiches befinden.

Die nachfolgende Beschreibung der GIF bei den Entwicklungsorganisationen, die sich vertieft mit Massnahmen auf diesem Gebiet befassen, soll dazu dienen, den Focus, die Bedeutung und den Rahmen dieser Massnahmen aufgrund des heutigen Standes darzustellen. Die Darstellungen beruhen auf Gesprächen mit Vertretern der Organisationen.

\subsection{Direktion für Entwicklungszusammenarbeit und humanitäre Hilfe (DEH)}

Im Rahmen der schweizerischen Entwicklungszusammenarbeit unterstützt die DEH namentlich auch die Industrialisierungsanstrengungen der Entwicklungsländer, wie dies im Bundesgesetz über Entwicklungszusammenarbeit und humanitäre Hilfe von 1976 festgehalten ist. Dort wird die Förderung von Handwerk und örtlicher Kleinindustrie als einer von fünf Schwerpunkten der Zusammenarbeit erwähnt. Die DEH sucht durch ihre GIF-Massnahmen folgende Ziele zu erreichen:

1. Förderung einer geographisch dezentralen und branchenmässig diversifizierten Industrialisierung.

2. Erhaltung eines hohen Anteils von Arbeitskräften in der Produktion der Unternehmen.

3. Verwendung lokaler Rohstoffe und angepasster technologischer Verfahren. 
4. Zur Erhöhung der nationalen Wertschöpfung und zur Versorgung des Binnenmarktes beitragen.

5. Einen Beitrag zur Verbesserung der Zahlungsbilanz leisten.

6. Individuelle technische und kaufmännische/unternehmerische Fähigkeiten fördern.

7. Integration der Massnahmen in bereits bestehende Strukturen und Prozesse.

Der DEH stehen für ihre Tätigkeit in diesem Sektor verschiedene Instrumente zur Verfügung. Sie kann eigene Projekte - in Zusammenarbeit mit Partnerorganisationen im Entwicklungsland - durchführen oder Projekte an andere Organisationen in Regie vergeben. Sie kann ausserdem eine Finanzhilfe an Projekte anderer Organisationen gewähren. Dabei kann es sich um Kofinanzierungen von Programmen internationaler Organisationen und von Entwicklungsbanken handeln oder um Programmkredite an schweizerische Entwicklungsorganisationen, welche bestimmte Aktionen in diesem Sektor einschliessen.

Die DEH beauftragt in diesem Sektor mit Regieprojekten hauptsächlich Swisscontact, Intercooperation und Helvetas. Einen Programmkredit, mit dem u.a. Projekte dieses Sektors gefördert werden, erhält Swissaid.

Kofinanzierungen für Projekte internationaler Organisationen werden z.B. an das BIT für die Förderung des städtischen, informellen Sektors in Afrika und an die Interamerikanische Entwicklungsbank für ihre Förderungsprogramme von Kleinunternehmern geleistet.

Der Umfang der finanziellen Mittel, welche die DEH für diese Aktionen aufwendet, beträgt durchschnittlich knapp unter $10 \%$ der jährlichen Ausgaben für bilaterale Aktionen und beläuft sich 1982-1985 z.B. auf 100,2 Mio Sfr. Die Finanzierung von Projekten, welche von Dritten durchgeführt werden, nimmt bei den Aktivitäten der DEH breiten Raum ein, wurden doch in der Periode 1982-85 von den 92 in diesem Sektor geförderten Projekte 55 in Regie vergeben.

Die von der DEH selber durchgeführten oder (teil)finanzierten Projekte weisen einen breiten Fächer möglicher Förderungsmassnahmen auf, die sich grundsätzlich in vier Ansätze gliedern lassen. Der erste, in der GIF zuerst angewandte und heute noch zentrale Ansatz ist jener spezifischer, formaler Berufsbildungsmassnahmen auf verschiedenen Stufen, die sich durch ein breites Angebot technischer und kaufmännischer Ausbildungsmöglichkeiten direkt an die zukünftigen oder bereits aktiven einzelnen Handwerker, Techniker und Kleinunternehmer richten.

Der zweite Ansatz richtet sich an die bereits bestehenden Betriebe und lässt diesen eine Reihe von Förderungsmassnahmen zukommen. Diese Betriebe können durch Einzelmassnahmen wie technische und kaufmännische Beratung, Vergabe von Kleinkrediten zu Investitionszwecken oder zur Ueberbrückung von Liquiditätsschwierigkeiten und Dienstleistungen bei Einkauf und Vermarktung der Produkte gefördert werden. Die Ausrichtung dieser Förderungsmassnahmen benötigt bei der Projektkonzeption und 
Durchführung eine zweifache Selektion: Zunächst müssen die Branchen, die gefördert werden sollen, ausgewählt werden und sodann die Betriebe innerhalb der Branchen. Je nach Strenge dieser Selektionskriterien wird die Zielgruppe der geförderten Unternehmen enger und homogener oder aber breiter und heterogener sein.

Der dritte Ansatz schliesst den zweiten indirekt ein, doch führt der Weg über die Förderung von Zusammenschlüssen innerhalb des Sektors, besonders von Selbsthilfeorganisationen des Handwerks und der Kleinindustrie. Der einzelne Unternehmer muss also Mitglied eines Verbandes werden, um in den Genuss von Förderungsmassnahmen gelangen zu können. Gemeinsame Projekte, wie z.B. Maschinennutzung, Materialeinkauf oder Preisgestaltung wollen dem Sektor und dem Einzelnen gleichzeitig zu Gute kommen.

Der vierte Ansatz schliesslich wird aus der Begrenzung der letzten zwei Ansätze sichtbar und nötig: Er zielt nicht auf die intrasektorielle Förderung, sondern auf die Verbesserung der Rahmenbedingungen für diesen Sektor durch die Gestaltung einer Wirtschaftspolitik, welche die Nutzung des wirtschaftlichen Potentials dieses Sektors durch staatliche Massnahmen besser zur Geltung kommen lassen will. Die Gestaltung dieser Rahmenbedingungen ist die Aufgabe der staatlichen Behörden des jeweiligen Enwicklungslandes, der bilaterale Donator kann bei der Realisierung seiner Projekts diese allenfalls beschränkt beeinflussen. Dieser letzte Ansatz geniesst in der schweizerischen Entwicklungszusammenarbeit keine Eigenständigkeit. Die Existenz vorteilhafter Rahmenbedingungen wird beim Entscheid zur Durchführung von Projekten in einem bestimmten Land in Betracht gezogen.

Eine wichtige Rolle bei der gegenseitigen Information und Koordination der Geberländer spielt ein informeller, jährlich ein-bis zweimal tagender Koordinationssausschuss (Steering Committee) der wichtigsten Geberländer und internationalen Organisationen im Handwerk- und Kleinindustriesektor.

Die von der DEH unterstützten Projekte befinden sich in 30 Entwicklungsländern, doch konzentrieren sich die Anstrengungen auf diejenigen unter ihnen, welche zur Gruppe der ärmsten Länder gehören und insbesondere auf jene ärmsten Länder, die zur Gruppe der 17 Schwerpunktländer der öffentlichen Entwicklungszusammenarbeit der Schweiz gehören. In Bezug auf die kontinentmässig zur Verfügung stehenden Mittel ist für den lateinamerikanischen Kontinent eine stärkere Berücksichtigung dieses Sektors bei der Mittelvergabe festzustellen. Die gesamten, für diesen Sektor ausgegebenen Mittel verteilen sich fast je zu einem Drittel auf die Kontinente Lateinamerika, Afrika und Asien. Die 92 von der DEH im Zeitraum von 1982-85 unterstützten Projekte umfassen folgende Aktivitäten :

- 45 Projekte mit Ausbildungs- und Weiterbildungsaktivitäten als Schwerpunkt.

- 37 produktionswirksame Projekte.

- 10 Projekte der sektorspezifischen Forschung und Entwicklung, sowie der Information und Dokumentation. 
Die bei der Durchführung der DEH-Projekte beteiligten Partnerorganisationen sind mehrheitlich staatliche und halböffentliche Institutionen des Bildungssektors, der industriellen Produktion und der Beratungsdienste.

Nach ersten, auf einige wenige Länder beschränkten Erfahrungen in der direkten Zusammenarbeit mit privaten Partnern beginnt diese erst in den letzten Jahren an Gewicht zu gewinnen, umfasst erst einzelne Projekte oder Kleinprojektprogramme und dient weiterhin dem Sammeln von Erfahrungen. Die volumenmässig bedeutenderen 39 Projekte (mit Ausgaben über 1 Mio Sfr. von 1982-85) wollen wir etwas näher betrachten.

Während sie sich der Betriebsgrösse nach gleichmässig zu je einem Drittel auf Handwerk, Klein- und Mittelindustrie verteilen, überwiegen regional gesehen die in Städten angesiedelten Projekte (22) gegenüber der Förderung von Projekten im ländlichen Bereich (17). Weiter ist die überwiegende Zahl der Projekte (33) im formalen Sektor angesiedelt, wir finden nur sechs im informellen Sektor. Sieben Projekte haben einen nationalen oder zumindest überregionalen Wirkungskreis, 16 einen regionalen und zwei beschränken diesen auf die dörfliche Ebene.

Den Schwerpunkt der bilateralen Zusammenarbeit in diesem Sektor bilden weiterhin die beiden Hauptaufgaben Ausbildung und Beratung, welche für etwa zwei Drittel der laufenden Projekte die jeweiligen Hauptaktivitäten bilden. Die Herstellung von Geräten und Maschinen zur handwerklichen und kleinindustriellen Produktion ist ein weiterer Schwerpunkt der DEH-Projekte.

Ins Gewicht fallen die Verbreitung neuer Herstellungstechniken und der Aufbau von Werkstätten und Kleinbetrieben. Schliesslich bildet die eigentliche Produktion von Gütern und die Unterstützung von Selbsthilfeorganisationen einen letzten Schwerpunkt der Entwicklungszusammenarbeit in diesem Sektor. Die Zielgruppen, welche durch die Projekte erreicht werden sollen, sind im ersten Ansatz, jenem der Berufsbildung, nicht genauer einzugrenzen, dient er doch dazu, spezifische Berufskenntnisse zu vermitteln, ohne auf besondere Zielgruppen ausgerichtet zu sein. Die traditionelle Ausrichtung auf formale technische Ausbildung - erst in den letzten Jahren ergänzt durch Ausbildungsaktivitäten im informellen Sektor - lässt allerdings eine Bevorzugung moderner Aktivitäten und eines engen Benutzerkreises vermuten, deren Kenntnisse nur ungenügend in den informellen Sektor diffundieren. Die Projekte, welche die produktiven Tätigkeiten des Sektors und den Aufbau seiner Organisationen unterstützen, lassen sich aufgrund der Wohn- und Arbeitssituation der Handwerker und Gewerbetreibenden und ihrer Produktion auf städtische und ländliche Bevölkerungsgruppen ausrichten. Einzelne Projekte suchen dabei gezielt die ärmeren Schichten der Städte oder die Handwerker auf dem Lande zu erreichen, die gleichzeitig Kleinbauern oder Landpächter geblieben sind. Kaum erreicht hingegen werden als spezifische Gruppe die Frauen durch die GIF der DEH. Eine Ausnahme bilden Projektkredite an die BID für spezifische Frauenförderungsprogramme in verschiedenen lateinamerikanischen Ländern.

Die Projektdurchführung wird bei den DEH-eigenen Projekten durch eigens rekrutierte Experten in Zusammenarbeit mit lokalen, von den Partner- 
organisationen zur Verfügung gestellten Mitarbeitern gewährleistet. Alle Projekte werden periodischen Evaluationen unterzogen, deren Methoden voneinander abweichen können. Am meisten zur Anwendung kommt die periodische Evaluation durch externe Experten und die permanente Projektbegleitung durch externe Berater. Zunehmendes Gewicht - in Ergänzung zu externen Evaluationen - erhalten eingebaute Evaluationen.

Die projektübergreifende Steuerung der Aktivitäten geschieht einerseits regional durch die Festlegung und Koordination im Rahmen der jeweiligen Länderprogramme und sektoriell durch den neu geschaffenen Fachdienst "Handwerk und Industrie». Diesem obliegt die Förderung und Sicherstellung einer fachlich qualifizierten Sektorpolitik und die interne Begleitung einzelner Projekte.

Schliesslich beteiligt sich die DEH an der Finanzierung zweier spezifischer Dienstleistungsstellen, welche die Aktivitäten in diesem Sektor unterstützen, die KODIS (Koordinations-, Dokumentations- und Informationsstelle für Berufsbildung in Entwicklungsländern, Winterthur) und die SKAT (Schweizerische Kontaktstelle für angepasste Technologie, St. Gallen). Die Konzeption der Hilfe hat sich aufgrund der Erfahrungen der Projekte und der Einschätzung des Sektors in den letzten Jahren stark verändert und unterliegt weiterhin einer laufenden Neubeurteilung. Das Schwergewicht soll sich von der Berufsausbildung auf produktive und organisationsfördernde Projekte verlagern.

Berufliche Grundlagenausbildung scheint zwar weiterhin nötig, um einem Land die nötigen Fachkräfte zur Verfügung zu stellen, sie kann jedoch kein breites, technisches Wissen vermitteln und kann eine direkte Förderung des Sektors nicht ersetzen.

Innerhalb des Sektors verlagert sich der Schwerpunkt der Förderung aus Gründen der Beschäftigungswirksamkeit auf kleine und dezentrale Unternehmen, auf eine umfassende Förderung der unternehmerischen Potentiale im kleinindustriellen und handwerklichen Bereich, auf die Integration mit anderen Entwicklungsmassnahmen und auf die Unterstützung von Selbsthilfegruppen. Damit verlagert sich auch das Profil der Partnerorganisationen, die weniger in staatlichen Produktions- oder Dienstleistungsbetrieben gesucht werden, sondern in lokalen privaten Organisationen und in den Selbsthilfsorganisationen des Gewerbes selbst.

\subsection{Swisscontact}

Swisscontact, Schweizerische Stiftung für technische Entwicklungszuammenarbeit, legt das Schwergewicht ihrer Arbeit auf den Aufbau und die Leitung von Lehrwerkstätten, ergänzt durch landtechnische Projekte, welche dem gemeinsamen Unterhalt und Einsatz von Landmaschinen dienen. Ausgeweitet werden diese Aktivitäten seit 1981 durch den Aufbau eines Gewerbeförderungsprogramms. Ausserdem werden auf Anfrage kurze Beratungsmissionen von «Senior Experts» organisiert.

Die Swisscontact verfolgt das Ziel, einen Beitrag zur Bildung einer Mittelschicht von qualifizierten Berufsleuten zu leisten und durch deren techni- 
sche Ausbildung die eigenständige Entwicklung der Länder zu begünstigen. Die Gewerbeförderung soll insbesondere die Gewerbetreibenden bei der Führung ihrer Kleinbetriebe unterstützen, bestehende gewerbliche Selbsthilfeorganisationen verstärken oder neue aufbauen, und zu einer Erhöhung des Einkommens beitragen, indem die Qualität der Arbeit verbessert und die Produktivität erhöht werden kann.

Das Programm der Gewerbeförderung kann selektiv in all jenen Ländern realisiert werden, in denen Swisscontact aufgrund ihrer Berufsbildungsprojekte bereits tätig ist oder war und wo deshalb Kontakte zu potentiell Begünstigten und eine technische Infrastruktur bestehen. Im Rahmen des Gewerbeförderungsprogramms wurden bisher erst seit 1981 in Costa Rica Erfahrungen gesammelt, neue Programme sind seit 1985 in Guatemala und in Sri Lanka im Aufbau, in Indonesien und Tunesien wurden 1986 Abklärungen durchgeführt.

Swisscontact verfügt im Rahmen dieses Programms über ein Budget von jährlich 0,5-0,7 Mio Sfr, welches für betriebliche Beratung, Dienstleistungen und einen Kreditfonds verwendet wird. Die Durchführung des Programms stützt sich auf eine Zusammenarbeit mit halbstaatlichen Organisationen und die im Laufe der Durchführung des Programms zu gründenden Selbsthilfeorganisationen ab.

Wenn auch in der Wahl der Länder durch die Aktivitäten der Swisscontact im Berufsbildungsbereich eine gewisse Vorselektion gegeben ist, müssen in einem Land weitere Voraussetzungen erfüllt sein, um in den Genuss einer Unterstützung zu kommen.

Es sind dies:

- Die Existenz von kleingewerblichen Reparaturbetrieben.

- Eine staatliche Ordnungspolitik, welche die Förderung der privaten Initiative und die Gründung von Selbsthilfegruppen erlaubt.

- Ein BSP mittlerer Höhe innerhalb der Skala der Entwicklungsländer und somit das Vorhandensein einer für die angebotenen Dienstleistungen gesicherten Nachfrage.

- Eine Ausrichtung des Programms auf Dienstleistungen im Unterhaltsbereich von technischen Produkten, in Anlehnung an die bereits realisierten Ausbildungstätigkeiten.

Als Projektpartner werden effizient arbeitende private Partner bevorzugt, doch kann, falls erforderlich, auch mit staatlichen oder halbstaatlichen Partnern zuammengearbeitet werden. Die Selbsthilfeorganisationen des geförderten Gewerbes sollen in die Projektträgerschaft integriert werden.

Die Verantwortung für die Projektdurchführung liegt vor Ort bei den von Swisscontact eingesetzten Experten, die entweder Projektleiter sind oder Beraterfunktion ausüben können. Für den Aufbau und die Durchführung der Projekte hat Swisscontact Richtlinien ausgearbeitet, die eine einheitliche Projektkonzeption erlauben sollen, ohne ins rezepthafte abzugleiten. Grosses Gewicht wird auf die Projektleitung im Feld gelegt, der eine hohe Eigenverantwortlichung mit grossen Entscheidungs- und Organisationskompetenzen innerhalb der Organisation zuerkannt wird. Bei den Pro- 
jekten mit einer grösseren Anzahl Begünstigten wird die Projektleitung durch regionale Berater verstärkt und durch Vertreter lokaler Selbsthilfegruppen, welche eine Gruppenleiterfunktion ausüben, ergänzt. Die Laufzeit der Projekte wird in 2-Jahresphasen unterteilt. Der Sachbearbeiter der Swisscontact unternimmt jährliche Projektreisen, welche der internen Evaluation dienen, und wird in der Projektbearbeitung durch einen Konsulenten unterstützt. Ausserdem besteht innerhalb der Swisscontact eine sektorspezifische Beratergruppe für das gesamte Gewerbeförderungsprogramm.

Die Zielgruppen der Gewerbeförderung sind die jeweiligen Betriebsinhaber der selektionierten Branchen. Bei der Selektion bildet die berufliche Qualifikation ein wichtiges Element. Die fehlende formale Ausbildung kann durch eine grosse praktische Erfahrung und hohe Motivation zur Weiterausbildung ausgeglichen werden. Regional können die zu fördernden Betriebe sowohl auf dem Lande als auch in der Stadt angesiedelt sein.

Im laufenden Gewerbeförderungsprojekt in Costa Rica geht es darum, die Qualität in der Reparatur und Wartung von Maschinen, Fahrzeugen und Geräten langfristig zu verbessern. Damit sollen die finanziellen Mittel und die technischen Leistungen der Kleinbetriebe verbessert und das Einkommen der Gewerbetreibenden erhöht werden. Der Projektpartner ist eine sich im Aufbau befindliche Organisation der kleinen Unternehmer.

Das Projekt erfasst verschiedene Mechanikergruppen, Monteure, Spengler und Schlosser; diese müssen selbst in ihrem Betrieb arbeiten, um gefördert werden zu können. Es umfasst Betriebsberatungen, materielle Dienstleistungen, Kreditvergebungen, Ausbildungskurse und den Aufbau der Kleinunternehmerorganisation «APTAMAI».

Das Gewerbeförderungsprogramm in Costa Rica wurde 1985 durch den das Projekt begleitenden Konsulenten evaluiert, eine externe Evaluation wurde bis jetzt noch nicht durchgeführt.

Die bisherigen Aktivitäten des Programms erbrachten sowohl auf der einzelbetrieblichen als auch auf der Ebene der Strukturierung des Sektors positive Resultate. Vom Projekt werden gegenwärtig ca. 200 Gewerbetreibende mit Dienstleistungen versorgt. Die im Laufe des Projekts gegründete APTAMAI hat teilweise bereits die Kompetenz erlangt, die Aktivitäten des Projektes selbst auszuführen.

Die Vergabe von kurzfristigen Krediten ermöglicht den Begünstigen, erstmals mit mehr Kapital zu arbeiten, da die Mitglieder der APTAMAI bisher bei den Banken nicht kreditwürdig sind. Die individuellen Beratungen erfolgen immer mehr auf Anfragen der Gewerbetreibenden selber und gehen kaum mehr von den Swisscontact-Experten aus.

Seit die Einzelberatung durch Erfahrungsaustausch und Gruppenarbeit ergänzt wird, erhöhte sich auch die Nachfrage nach diesen Dienstleistungen und die Mitgliederzahl von APTAMAI. Die Arbeit in Erfahrungsgruppen und die Weiterbildung in spezifischen Berufsgruppen entspricht einem grossen Bedürfnis und fördert den Aufbau von berufsspezifischen Selbsthilfeorganisationen. 
Bei wachsendem Kundenkreis wird die Selektion der Mitglieder, die leistungsfähige Dienstleistungen erbringen sollen, anspruchsvoll. Das Ziel, qualitative und finanzielle Verbesserungen im Kleinbetrieb zu erreichen, wird schwieriger erreichbar. Die Auswirkungen des Projekts auf die Bevölkerung wurden bisher nicht beurteilt. Sicher hat die Versorgung mit technischen Dienstleistungen im Kundenkreis der Kleinunternehmer zugenommen. Eine Beeinflussung der wirtschaftspolitischen Rahmenbedingungen des Sektors durch seine Trägerschaft ist jedoch wegen ihrer geringen politischen Bedeutung kaum realisierbar und wird auch nicht angestrebt.

Das Förderungskonzept von Swisscontact erhielt aufgrund der in Costa Rica gemachten Erfahrungen gewisse Aenderungen und sieht jetzt eine gleichzeitige Förderung der Einzelbetriebe und einen Aufbau der Selbsthilfegruppen vor. Die rein individuelle Betriebsberatung stösst rasch an ihre Grenzen, auch wenn sie im Einzelfalle wirksam ist, und reicht nicht aus, um einen ganzen Sektor zu strukturieren.

\subsection{Swissaid}

Swissaid, Schweizerische Stiftung für Entwicklungszusammenarbeit, setzt sich für eine Verbesserung der Lebensbedingungen der ärmeren Schichten in ländlichen Gebieten der Dritten Welt ein und unterstützt dazu in erster Linie Selbsthilfeprojekte, welche diesen Schichten direkt zugute kommen.

Innerhalb der Projektarbeit der Swissaid kann der Sektor Handwerk und Kleinindustrie allein oder integriert in multisektoriellen Projekten gefördert werden. Diese Förderung orientiert sich an den folgenden Zielen :

1. Schaffung von Arbeitsplätzen in ländlichen Gebieten ausserhalb der landwirtschaftlichen Produktion, insbesondere für Jugendliche.

2. Förderung einer dezentralen, kleinindustriellen Produktion und Wartung und einer ausgeglichenen wirtschaftlichen Entwicklung.

3. Förderung von Produkten für den nationalen Markt und Ermöglichung einer Importsubstitution.

4. Gebrauch von Technologien, welche von den Produzenten rasch beherrscht werden können und keine grössere Abhängigkeit vom Ausland entstehen lassen.

5. Nutzung von lokalen menschlichen Ressourcen und Rohstoffen.

6. Ermöglichung eines technischen und betriebswirtschaftlichen Wissenstransfers.

Gefördert wird dieser Sektor innerhalb der 10 Länder, in denen Swissaid tätig ist, hauptsächlich in Indien und in den in diesem Jahrzehnt als Partnerländer gewählten Ländern Guinea-Bissau, Zimbabwe und Nicaragua. Die im Jahr 1985 für die Unterstützung dieses Sektors bewilligten Mittel betrugen 473.000 Sfr, was 9\% der gesamten, für die Projektarbeit im Ausland bewilligten Mittel ausmacht. Als Instrument zur Erreichung der Förderungsziele setzt Swissaid eine projektgebundene Finanzhilfe ein, welche an nationale Projektpartner ausgerichtet, vom Schweizer Sekretariat und von einem 
nationalen Koordinator betreut wird. Die Initiative zu Projektanträgen an die Swissaid kommt dabei nicht vom Sekretariat, sondern von nationalen Projektpartnern und wird über den nationalen Koordinator der Geschäftsstelle und schliesslich dem Stiftungsrat zum Entscheid vorgelegt. Die DEH, welche die Swissaid durch einen Programmkredit unterstützt, prüft das Projekt ebenfalls, falls es aus diesem Programm-Kredit kofinanziert werden soll. Eine Finanzierungsphase erstreckt sich über jeweils ein bis zwei Jahre, eine längere Zusammenarbeit kann aber dem Partner informell zum voraus zugesichert werden. Die Finanzhilfe der Swissaid wird nur ausgerichtet, wenn der Projektpartner und/oder die Zielgruppe einen eigenen Beitrag zur Projektrealisierung leistet. Die konkrete Gestaltung einer Finanzhilfe soll im weiteren am Beispiel der GIF-Förderung in Guinea-Bissau durch Swissaid erläutert werden.

Unterstützt werden Projekte, welche den traditionellen, ländlichen Handwerksektor und die Verarbeitung von Agrarprodukten oder neue kleinindustrielle Betriebe auf dem Lande zum Ziel haben.

Die Produktion kann dabei individuell, in produktionsorientierten Arbeitsgruppen auf dörflicher Ebene oder im Kleinbetrieb geschehen.

Die Projekte sollen eine Verbesserung der Produktionstechnik der Verarbeitung und Vermarktung erlauben und eine Ergänzung zur traditionellen Produktion darstellen. Damit sollen interne Wirtschaftskreisläufe verstärkt und der entwicklungspolitische Gesamtrahmen nach Möglichkeit beeinflusst werden.

Die Finanzhilfe der Swissaid wird im allgemeinen für folgende Aktivitäten verwendet:

1. Einkauf von Produktionssmitteln (Rohstoffe, Werkzeuge, Geräte) und zwar als Geschenk, Kredit oder aus einem Rotationsfonds finanziert.

2. Finanzierung der beruflichen Weiterbildung (Alphabetisierungskurse, technische und betriebswirtschaftliche Weiterbildung der Zielgruppen).

3. Erschliessung des Absatzmarktes (Uebernahme der Kosten für lokales Beratungs- und Administrationspersonal).

Die Projektpartner sind operationell tätige, staatliche oder private Institutionen. Die staatlichen Institutionen - in Guinea-Bissau das Ministerium für Industrie und das Ministerium für ländliche Entwicklung - werden oft durch ausländische Entwicklungshelfer verstärkt; die privaten Partner - in Guinea-Bissau ausländische NGOs - können auch Entwicklungshelfer zur Verfügung stellen. Die wichtige Rolle ausländischer Fachleute ist in der Arbeit von Swissaid ein Sonderfall und auf den akuten Kadermangel in Guinea-Bissau zurückzuführen. Diese Partner führen eigentlich die Projekte vor Ort durch und arbeiten mit den Zielgruppen zusammen.

Die Zielgruppe umfasst möglichst alle Handwerker einer Region. Eine individuelle Selektion ergibt sich aus deren Motivation, sich an der Förderung zu beteiligen.

Von Swissaid selbst werden die finanzierten Projekte durch den Koordinator vor Ort und durch jährliche Länderbesuche des Sachbearbeiters begleitet und evaluiert. Hauptinstrumente sind dabei die Projektbesuche 
und Berichte. Die Länderbesuche dienen der Identifikation neuer Projekte, der Kontrolle über die Mittelverwendung und der inhaltlichen Begleitung und eventuellen Neuorientierung der Projekte. Eine weitere Aufgabe der Besuche liegt in der Evaluierung der Partner von Swissaid.

Finanz- und Arbeitsberichte müssen mindestens einmal jährlich verfasst werden. Die Zusammenarbeit zwischen dem Partner und Swissaid ist vertraglich geregelt.

Die dreijährigen Aktivitäten der Swissaid in spezifischen Regionen von Guinea-Bissau haben eine Anzahl positiv zu würdigender Effekte hervorgerufen, die eine Erreichung der Förderungsziele als realistisch erscheinen lassen.

Durch die Förderung konnte eine Stärkung der wirtschaftlichen Struktur der Regionen erreicht werden, insbesondere durch eine relativ signifikante Wirkung der Förderungsmassnahmen auf die Produktionshöhe und Qualität des dörflichen Handwerks. Die verbesserte Produktionssituation kann zu einer vermehrten Nutzung vorhandener Rohstoffe führen, die endgültige oder saisonale Abwanderung der Handwerker bremsen und einen Beitrag gegen den Zerfall des landwirtschaftlichen Sektors leisten.

Diese Stabilisierung handwerklicher Aktivitäten führt ansatzweise zu einer verbesserten Lebenssituation der Bevölkerung im Projektgebiet, durch eine verbesserte Beschäftigungs- und Einkommenssituation, und zur Stärkung der familiären Strukturen durch abnehmende Migration.

Die bisherigen Erfahrungen in der Realisierung dieses Programms bestärken die Prognose über das vorhandene Potential an regionalen Investitions- und Absatzmöglichkeiten, bestätigen die Schwierigkeiten beim Import notwendiger Rohstoffe und Werkzeuge wegen einer mangelhaften Abwicklung des staatlich reglementierten Aussenhandels, sowie den dominierenden Einfluss der Nachbarökonomie Senegals auf die Region (z.B. durch das höhere Preisniveau und breitere Sortimente).

Mangelnde technische und animatorische Qualifikation der mit der Durchführung des Programms betreuten einheimischen Kader verlangsamen die Wirkung der Förderungsmassnahmen.

Das Beispiel Guinea-Bissau zeigt auch, dass die Konzeption der GIF der Swissaid breiter geworden ist. Zudem spielte die GIF volumenmässig früher eine geringere Rolle als heute. In den 70er Jahren stand die Förderung der beruflichen, d.h. der technischen und kaufmännischen Ausbildung von Mitgliedern aus Basisgruppen im Vordergrund. Solche Ausbildungsprojekte werden von Swissaid weiterhin unterstützt, auch in Guinea-Bissau. Darüber hinaus wird der einzelne Handwerker mit einem Bündel verschiedener Massnahmen unterstützt, welche seine Produktion auf verschiedenen Stufen beeinflussen, und durch den Zusammenschluss der einzelnen Produzenten soll eine Stärkung ihrer Marktposition erreicht werden. 


\subsection{FUNDES}

FUNDES, Stiftung für ökonomische und soziale Entwicklung, ist eine privatwirtschaftliche Initiative zur Förderung von Gewerbe und Kleinindustrie in der Dritten Welt, insbesondere in Lateinamerika. Die Dynamisierung und Stabilisierung dieses in jeder volkswirtschaftlichen Struktur vorhandenen kleinindustriellen und gewerblichen Sektors, der aber nur ungenügend mit den notwendigen Produktionsfaktoren ausgestattet ist, ist in ausgewählten Ländern Ziel der Stiftung. Sie gibt damit der Gründung und Entwicklung von Kleinunternehmen und dadurch der Schaffung von Arbeitsplätzen und Einkommen im kleinindustriellen und Dienstleistungssektor Priorität. Für diese Prioritäten sprechen, dass die Aufnahmefähigkeit des landwirtschaftlichen Sektors für zusätzliche Arbeitnehmer mittelfristig begrenzt ist und zudem bis heute wesentlich mehr Hilfsgelder in diesen Sektor geleitet wurden. In den lateinamerikanischen Volkswirtschaften ist der handwerkliche und kleinindustrielle Sektor heute schwach ausgebildet, muss aber in Zukunft bei der Versorgung der Bevölkerung mit Gütern und Dienstleistungen und bei der Schaffung von Arbeitsplätzen und Einkommen eine dynamischere Rolle spielen.

Zur Erreichung dieser Ziele arbeitet die Stiftung grundsätzlich mit drei verschiedenen, sich ergänzenden Instrumenten:

- Die Schaffung und Finanzierung eines Bürgschaftsfonds, welcher den Mitgliedern der Zielgruppen den Zugang zu kommerziellen Bankkrediten erleichtern soll, da er das Risiko des kreditgebenden Finanzinstituts gegenüber dem Gewerbetreibenden vermindert. Kreditgeber selbst sollen möglichst wiederum lokal oder regional tätige Finanzinstitutionen sein.

- Die Beratung des einzelnen Geförderten umfasst generell die organisatorisch/administrativen und technischen Aufgaben seines Unternehmens und kann spezifisch vertieft werden.

- Die Bildung von Erfahrungsgruppen aus den Reihen der einzelnen Kleinunternehmer soll dem Erfahrungs- und Wissensaustausch dienen und das Selbstverständnis der Kleinunternehmer fördern.

Die konkrete Ausgestaltung dieser Instrumente passt sich dem nationalen Umfeld an und wird durch nationale FUNDES-Stiftungen bewerkstelligt.

FUNDES selbst ist mit einzelnen nationalen Stiftungen föderalistisch organisiert. FUNDES-Schweiz stiftet einen Teil des zur Tätigkeit im ausgewählten Entwicklungsland nötigen Kapitals, welches vom Stiftungskomitee der nationalen FUNDES-Stiftung verwaltet wird. In diesem Stiftungskomitee sind Vertreter der FUNDES-Schweiz in der Minderheit. Sie gewähren in der Regel der nationalen Stiftung eine technische Beratung und führen mit dem Stiftungskomitee eine laufende Evaluation seiner Tätigkeit durch. Das nationale Komitee arbeitet für die Beratung und Ausbildung mit lokalen Beratern zusammen, die sich z.B. aus Vertretern des Unternehmerverbandes zusammensetzen. Die jeweiligen Stiftungen verfügen über ein Sekretariat. 
Bearbeitet werden Projekte aus dem Sektor des Handwerks und der Kleinindustrie (mit bis zu ca. 10 Arbeitern). Die Substanz des Bürgschaftsfonds muss dabei erhalten bleiben und Kreditverluste und administrative Kosten müsen über den Zinsertrag des Fonds finanziert werden.

Der durch den Bürgschaftsfonds ermöglichte Kredit, max. 20.000 Dollar, wird von einer mit FUNDES zusammenarbeitenden Bank nach einem Gespräch mit dem Kreditnehmer und einer betriebswirtschaftlichen Begutachtung innert kurzer Frist (ca. 20 Tage) gewährt.

Ueber eine individuelle Beratung hinausgehend besteht ein Kursangebot, welches die Kleinunternehmen mit kaufmännischem Grundwissen bekanntmacht. Die Zusammenarbeitsform mit den Begünstigten soll individuell bleiben, um die Beratung möglichst offen und vertrauensfördernd, und die Förderung möglichst effizient gestalten zu können.

FUNDES begann zuerst in Panama und wird seine Tätigkeit nach dem gleichen Organisationsmodell ab 1987 nach Costa Rica und Guatemala ausdehnen. Panama wurde als Pilotland gewählt, um das Modell in einer als schwierig eingeschätzten Praxis testen zu können. Zwar verfügt das Land über ein relativ hohes BSP (1984: 1980 US-Dollar), doch ist es durch eine duale Wirtschaftsstruktur und eine schwach ausgebildete Handwerksstruktur und Kleinindustrie gekennzeichnet. Panama verfügt kaum über einen produzierenden Gewerbesektor, und die Banken gaben früher Unternehmen aus diesem Sektor keinen Kredit. Die bisherigen Erfahrungen - abgesehen von der Anzahl der geleisteten Bürgschaften (in den ersten anderthalb Jahren der Tätigkeit wurden ca. für 100 Betriebe Kredite ermöglicht und Beratungen erbracht) zeigt, dass mit der von FUNDES gewählten Strategie der Zugang zu Bankkrediten geschaffen wird. Mittelfristig wird die Strategie nach Meinung von FUNDES jedoch nur erfolgreich sein, wenn sich der Kleinunternehmer im Markt behaupten kann, seine Beratung selbst finanziert und ohne Unterstützung von FUNDES den Zugang zu - für ihn akzeptablen Bankkrediten findet. Interessenten an Bürgschaften sind vorhanden, diese jedoch zur Teilnahme in den Erfahrungsgruppen zu aktivieren, benötigt einen längerfristigen Prozess.

Eine für Mitte 1987 geplante Evaluation des FUNDES-Programms in Panama soll die Frage nach der Projektqualität und die betriebswirtschaftliche Analyse der geförderten Kleinunternehmer zum Inhalt haben. Die Wirkung der Strategie von FUNDES hängt vom Ausmass und der Entwicklungsfähigkeit des unternehmerischen Denkens und Handelns (Risikobereitschaft, Einsatz und Können) der geförderten Unternehmer ab.

\subsection{UNIDO - Investment-Promotionsbüro/Zürich}

Die UN-Organisation für industrielle Entwicklung UNIDO hat generell zum Ziel, die Industrialisierung der Entwicklungsländer durch Beachtung marktwirtschaftlicher Prinzipien und in Kooperation mit der Privatwirtschaft zu fördern. 
Hauptsächliche Schwerpunkte ihrer Aktivitäten sind die Ausarbeitung nationaler Industrialisierungspläne, die Unterstützung einzelner Investitionen und die Aus- und Weiterbildung von Industriekadern. Die UNIDO eröffnete zu diesem Zweck in fünf westeuropäische Industrieländern sowie in Japan, den Vereinigten Staaten und in Polen spezielle Investitionsförderungsbüros.

Das 1978 eröffnete UNIDO-Investitionsbüro in Zürich hat zum Ziel, die Zusammenarbeit zwischen der Schweizer Industrie und Industriellen in Entwicklungsländern zu fördern und durch diese Zusammenarbeit industrielle Investitionen in den Entwicklungsländern zu ermöglichen. Diese Zusammenarbeit soll dabei kommerziell orientiert und mit einem beidseitigen finanziellen Risiko verbunden sein.

Das Büro wird durch einen Posten aus dem Kredit für handels- und wirtschaftspolitische Massnahmen im Rahmen der schweizerischen Entwicklungszusammenarbeit finanziert. Dieser Kredit wird vom BAWI verwaltet. Das laufende Budget beträgt 3,3 Mio Sfr für eine fünfjährige Tätigkeit des Büros. Die Ziele der mit diesem Kredit geförderten Massnahmen werden generell umschrieben als Beitrag zur breiten Erhöhung des Lebensstandards in verschiedenen Gruppen von Entwicklungsländern, sei es durch gesamtwirtschaftliche Effekte oder durch die Bevorzugung ärmerer Schichten, ohne dass die einzelne Massnahme direkte Auswirkungen auf bestimmte Bevölkerungsgruppen haben muss. Die Formen der Zusammenarbeit, welche das Büro den Investoren vorschlagen kann, reichen von technischen Beratungsverträgen über eine finanzielle Beteiligung und Ausbildungsprogrammen bis zu gemeinsamen neuen oder restrukturierten Unternehmen.

Die Instrumente, welche dem Büro in Zürich zur Verfügung stehen, sind:

1. Informationsvermittlung und Kontaktschaffung.

2. Kreditfazilität für Abklärungsstudien.

3. Absatzförderung.

4. Ausbildung und Beratung.

Die Informationsvermittlung und Kontaktschaffung ermöglicht der Schweizer Industrie und ihren Organisationen den Zugang zu Investitionsangeboten aus Entwicklungsländern. Diese gelangen über die UNIDO-Kanäle oder als direkte Anfragen ans Büro nach Zürich. Sie kann verstärkt werden durch die Organisation von mehrtätigen Besuchsprogrammen für Industrielle aus Entwicklungsländern bei ausgewählten Schweizer Unternehmen. Die Kreditfazilität wird weiter unten ausführlicher beschrieben. Die Absatzförderung für die Vermarktung von Produkten unterstützter Investitionen auf dem europäischen Markt befindet sich im Aufbau und wird gemeinsam mit der UNCTAD/GATT-Organisation "ITC» (International Trade Center) durchgeführt werden.

Die Ausbildung und Beratung beschränkt sich auf die Vermittlung von Weiterbildungsmöglichkeiten für Industrielle aus Entwicklungsländern in Schweizer Unternehmen - das UNIDO-Büro übertrug diese Aufgabe dem SIDI (Swiss Industrial Development Institute) - und auf die Beratung von 
derzeit zwei nationalen Investitionsförderungsbüros (China und Tunesien).

Die durch die Informationsvermittlung und Kreditfazilität zu fördernden Projekte bewegen sich auf einem Investitionsvolumen von 1-8 Mio Sfr. Kleinere Projekte sind finanziell uninteressant, da sie die entstehenden Kosten für den Investor nicht zu decken vermögen. Damit wird der eigentliche Handwerk- und Kleinindustriesektor nicht in die Förderung mit eingeschlossen, und Industrieinvestitionen mittlerer Grösse bilden das zu fördernde Segment. Gefördert werden können Projekte aus dem gesamten Industriebereich, andere Sektoren sind ausgeschlossen. Die Leistungen des schweizerischen Investors sollen dabei über eine blosse Kapitalbeteiligung hinausgehen und die Beteiligung oder Beratung der Geschäftsleitung und eine technische und betriebswirtschaftliche Ausbildung der Mitarbeiter umfassen.

Der Absatz der Produktion kann über den nationalen Markt hinaus auf den Export ausgerichtet werden, insbesondere um die für den Kauf und Unterhalt der Maschinen sowie die für die Produktion nötigen Devisen selber erarbeiten zu können.

Die einzelnen Projekte sollen den Industrialisierungsplänen der Gastländer entsprechen, über einen Erfolg wird schliesslich ihre Rentabilität entscheiden. Zur Vorbereitung der Investitionen steht ein Kredit-Fazilitätsfonds zur Verfügung, aus welchem potentielle Investoren bis zur Hälfte der Kosten für eine Abklärungsstudie finanzieren lassen können. Die restlichen Kosten trägt der Investor. Der schweizerische Investor muss dabei einen Umsatz pro Jahr von weniger als 500 Mio Fr. aufweisen. Wird eine Investition realisiert, ist dieser Kredit zurückzuerstatten. Projekte, welche aus diesem Fonds finanziert werden, erlauben dem Büro eine über die blosse Informationsvermittlung hinausgehende Begleitung des Projektes, z.B. hinsichtlich der Technologiewahl, bis zu einem Vertragsabschluss zwischen den Partnern. Die Fazilität steht nur für Investitionsvorhaben in Ländern mit einem BSP pro Kopf bis zu 1430 Dollar zur Verfügung.

Es gibt bei der Promotion keine Prioritäten oder Einschränkungen auf bestimmte Entwicklungsländer, ausser bei der Gewährung der Kreditfazilität. Die Zielgruppen sind nationale Industrielle aus den Entwicklungsländern und schweizerische Industrielle, welche zu Beginn der Kooperation mit der UNIDO verhandeln, danach aber selbständig weiterarbeiten.

Eine Begleitung der Investitionsvorhaben ist nur in einer ersten Phase möglich, wenn unter Organisation der UNIDO Kontaktgespräche stattfinden, und bei der Beurteilung eines Investitionsvorhabens im Rahmen der Kreditfazilität. Danach erfolgt die Zusammenarbeit allein zwischen den beiden Partnern. Die UNIDO kann höchstens eine technische Begleitung der Investition vorsehen. Im Rahmen der allgemeinen Informationsvermittlung konnten bis Ende 198542 Investionen mit einem Gesamtvolumen von 125 Mio Dollar bearbeitet werden. Im Rahmen der Kreditfazilität wurden $18 \mathrm{Kre}$ dite vergeben. Diese führten z.B. zu Abklärungsstudien über: Produktion von Rikschas in Bangla Desh, Herstellung von Aktivkohle in Pakistan, Kautschukverarbeitung in Sri Lanka, Herstellung von Apparaten zur Stromvertei- 
lung im Senegal, Sperrholzproduktion in Ecuador.

Die Tätigkeit des Büros selbst wurde 1984 nach Ablauf einer zweiten Arbeitsphase einer Evaluation unterzogen, in welchem die Instrumente des Büros und ihr Erfolg ausgewertet wurden.

Seine Aufgabe hat sich seit der Eröffnung konzeptionell nicht verändert; sie wurde durch die Schaffung der Kreditfazilität erweitert. Im Laufe der Jahre konnte der Bekanntheitsgrad erhöht werden, was zu vermehrten direkten Kontakten von Industriellen mit dem Büro führte. Soweit möglich wird eine intensivere Begleitung der Anfragen und Projekte in ihrer Anfangsphase angestrebt, doch muss dies von den eigentlichen Projektpartnern auch gewünscht werden.

Eine stärkere Investitionsförderung stösst an entwicklungspolitische Grenzen, da viele Entwicklungsländer aufgrund ihrer wirtschaftlichen Situation grosse Investitionsrisiken in sich bergen, die Rentabilität der einzelnen Investition aber so gut als möglich gesichert sein soll, und schliesslich zu deren Realisierung ein vertrauenswürdiger, starker Partner im Entwicklungsland identifiziert werden muss.

\subsection{Profile und Beziehungen}

\section{Profilmatrix (vgl. Schema 4)}

Bei Vergleichen stellt sich zunächst die Frage nach den Aehnlichkeiten und Verschiedenheiten zwischen den Entwicklungsorganisationen. Mit dem Erstellen einer Matrix ist hier der Versuch unternommen worden, die Profile der befragten Organisationen anhand von sechs Kriteriensätzen darzustellen. Durch einen Vergleich der auf dieser Matrix sichtbaren Profile der einzelnen Organisationen ergibt sich eine wichtige Feststellung: Auf dem Gebiet der GIF führen unterschiedliche Organisationen mit unterschiedlichen Arbeitsmethoden unterschiedliche Aktionen durch, wobei es zwischen innen wohl teilweise Aehnlichkeiten, aber keine Gleichheiten gibt.

Im Anschluss an diese keineswegs triviale Aussage stehen drei Fragen im Vordergrund:

Sind die Unterschiede in den Ansatzpunkten, Arbeitsmethoden, Organisationsformen usw. der Förderung eine Antwort auf sehr verschiedene Situationen und Bedürfnisse in den Partnerländern? Die Vielfalt der Erscheinungsformen im GIF-Bereich würde konsequenterweise eine Vielfalt auf der Promotionsebene hervorrufen. Diese Erklärung passt zu der im ersten Teil beschriebenen Heterogenität des GI-Bereichs. Als Folge dieser Vielfalt spezialisieren sich einzelne Organisationen auf Teilaspekte und es ergibt sich eine Arbeitsteilung, die, soweit öffentliche Mittel über die DEH zu andern Organisationen kanalisiert werden, durchaus abgesprochen ist.

Sind die aufgeführten Unterschiede ein Abbild divergierender Auffassungen über die GIF in der Schweiz? In einer pluralistischen Gesellschaft können sich als Resultat von verschiedenen Vorstellungen und Wahrnehmungen der Entwicklungsmöglichkeiten des GI-Sektors verschiedene Ant- 


\section{Profilmatrlx der Förderorganisationen}

Schema 4

1. Soziale Priorität

Hilfeorientiert,

Umverteilung

2. Kleinprojekte $<0.5$ Mio Str/Projekt Projekt Gesamtaufwand

3. Verbände- und Institutlonentörderung Kollektive Selbsthilfe, Selbstorganisation

\section{Ruraler Sektor} Komplementarität zur Landwirtschatt

5. Leichte, stützende Interventionen Verbesserung von Vorund Randbedingungen, Information, Begleitung, wenig Personal vor Ort.

6. Breites Leistungsangebot

Viele Instrumente, viele verschiedene Aktionen

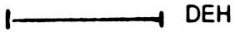

1.- - - - - Swissaid

_....... Swisscontact

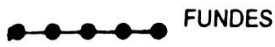

If.......... UNIDO-ZH

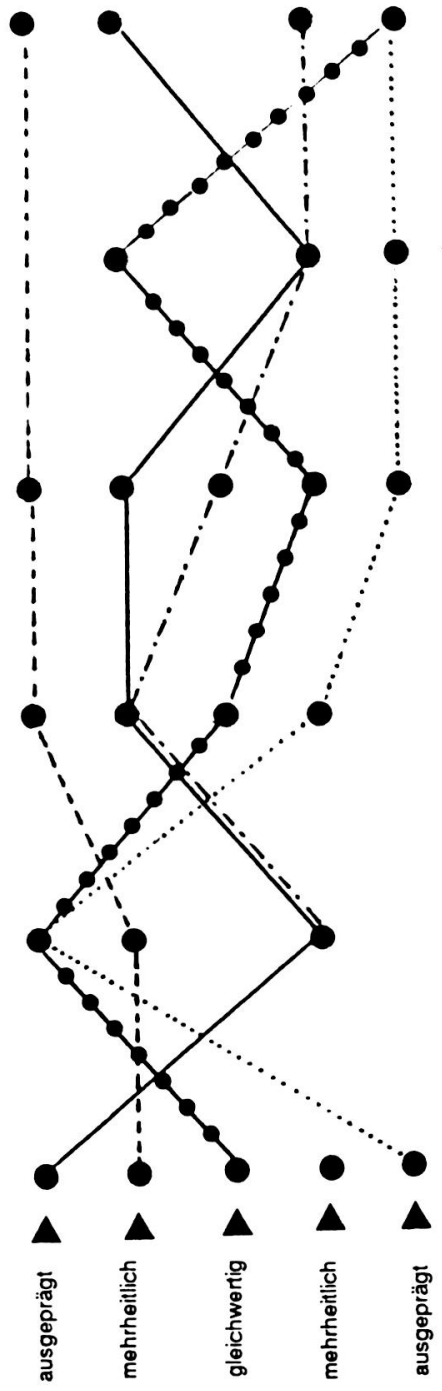

Markt-Prloritàt

Rentabilitätsorientient

Mittlere und grosse Projekte $>0,5$ Mio Str/Projekt

Gesamtaufwand

Unternehmer- und Elnzelbetrlebsförderung Persönliche Verantwortung. Initiative und Kompetenz, Konkurrenz

Urbaner Sektor

Versorgung städt. Bevölkerung min Dienstleistungen und Gütern.

Starke, durchführende Interventionen

Projektleitungen, eigenes Personal vor Ort 
worten bei GIF-Operationen ergeben. Auch diese Erklärung ist plausibel. Beispielsweise ermöglicht die homogene Trägerschaft der FUNDES-CH es der Stiftung, eine konzise, den Vorstellungen der Trägerschaft entsprechende Förderungsstrategie zu verfolgen. Dabei wird selbstverständlich der Privatinitiative dynamischer Unternehmerpersönlichkeiten viel Gewicht beigemessen. Auf der andern Seite zwingt der öffentliche Auftrag der DEH diese zu einer breiten Palette von Interventionen innerhalb des flexiblen Rahmens, der durch das Bundesgesetz vorgegeben ist.

Sind die Unterschiede in den Zielen, Strategien und Massnahmen Ausdruck von bisher fehlenden oder ungenügenden Erfahrungen über das, was in 'der GIF wirklich Bestand und Zukunft hat? Die eigenen Erfahrungen der schweizerischen Organisationen sind um die 25 Jahre jung und dort, wo es um Massnahmen in den schwierigen informellen/nicht strukturierten Untersektoren geht, erst wenige Jahre alt. Demnach hätte diese Situation noch zu keinen sicheren Lehren geführt, wodurch jede Organisation auf ihre eigene Strategie und Politik schwören kann. Tatsächlich ist das Bedürfnis nach Erfahrungsaustausch und Koordination auf nationaler und internationaler Ebene gross, sodass auch diese Teilerklärung möglich ist.

\section{Beziehungsgeflecht (vgl. Schema 5)}

Die Verschiedenheit und gleichzeitig die partielle Aehnlichkeit der Organisationen kommt ebenfalls im aufgeführten Beziehungsnetz der Organisationen zum Ausdruck. Ihr je verschiedener sozialer und politischer Ort, die unterschiedliche Organisation ihrer Entwicklungszusammenarbeit und unterschiedliche Zielgruppen ergeben ein Netz konzeptueller und finanzieller Beziehungen, das die Heterogenität und die Aehnlichkeit der Förderung zum Ausdruck bringt.

Wir haben bei der Aufzeichnung von Beziehungen zwischen den Institutionen fünf Bereiche unterschieden; sie ergeben sich aus den verschiedenen Hauptfunktionen, die von den Institutionen wahrgenommen werden: Finanzierung, Trägerschaft in der Schweiz, spezifische Hilfsfunktionen (wie fachspezifische Beratung, Dokumentation, externe Beurteilung), intermediäre Organisationen und Transmissionsstrukturen in den Empfängerländern und die Empfänger, die Zielgruppen, die Begünstigten selber. Die Abgrenzungen sind nicht immer eindeutig, besonders zwischen den letzten zwei Bereichen.

Das Schema macht einige präferentielle Kooperationslinien im Bereich der GIF sichtbar :

- Oeffentliche Mittel gehen in die DEH und das Zürcher UNIDO-Büro. Ein Teil der Mittel für die DEH wird an Regieträger und in Form von Beiträgen an eigene Projekte privater Organisationen weitergeleitet. Partner der DEH sind in den Empfängerländern primär staatliche und halbstaatliche Organismen und Entwicklungsbanken.

- Eine starke Verbindung besteht zwischen Swissaid, NGOs im intermediären Bereich und Basisorganisationen. 


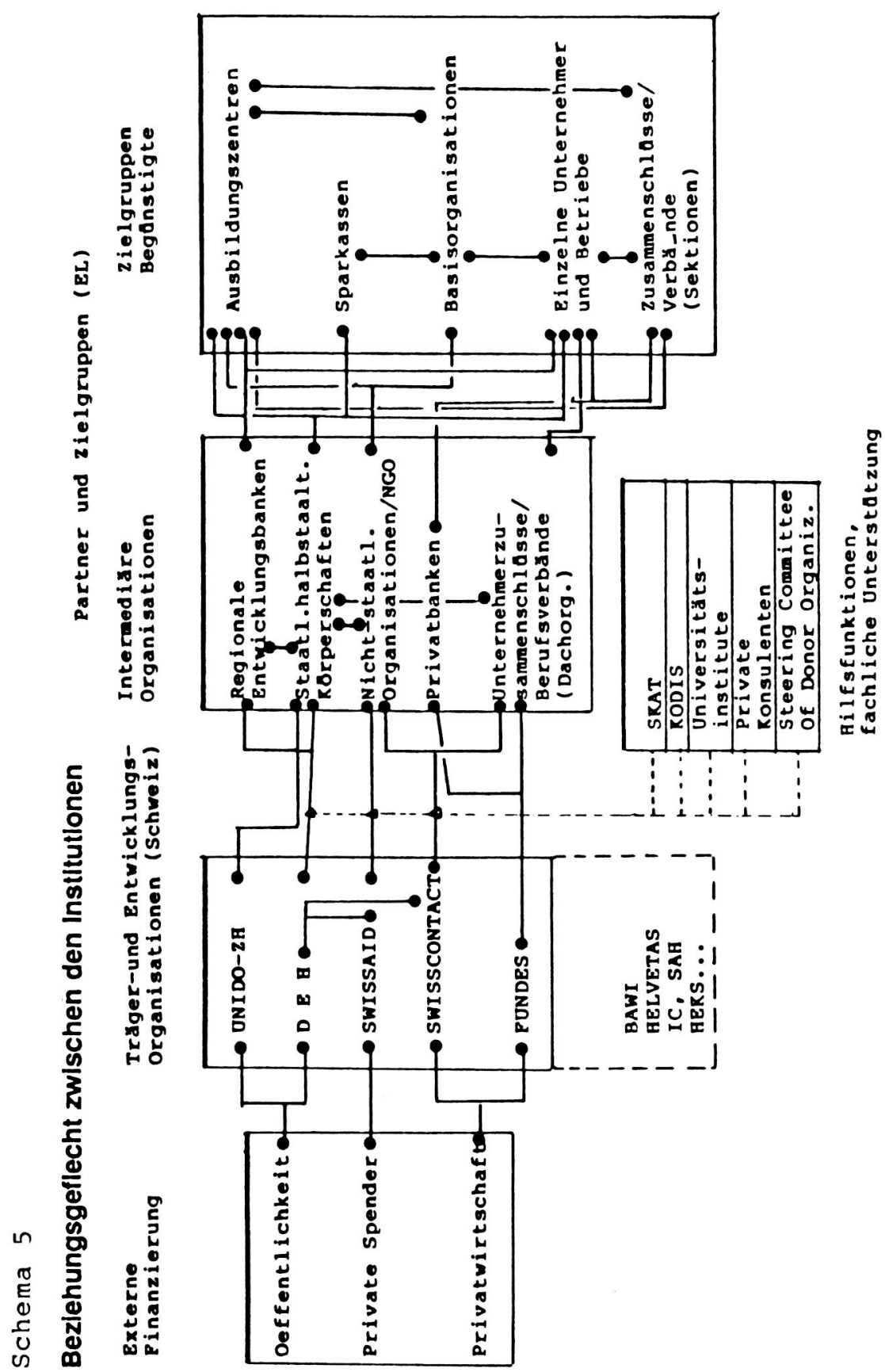


- Mittel der Privatwirtschaft fliessen zu Swisscontact und zur Stiftung FUNDES. Eine wichtige Linie geht von Swisscontact über staatliche und private Partner zur Berufsbildung. Die Partner von FUNDES sind Privatbanken und Unternehmergruppierungen, über welche vor allem Einzelbetriebe gefördert werden.

- Vielfache Beziehungen haben die intermediären Organisationen untereinander; dasselbe gilt auch für die verschiedenen Empfängergruppen. Aber auch zwischen den beiden Bereichen - intermediäre und Empfängerorganisationen - besteht ein dichtes Netz von Kooperationsbeziehungen. Zwar haben sich viele der intermediären Organisationen auf bestimmte Leistungen spezialisiert und unterhalten privilegiert Beziehungen mit einer oder mehreren Klientengruppen. Aber es besteht eine Vielzahl von Organisationen in beiden Bereichen, sodass die Zusammenarbeit (zumindest theoretisch) in irgend einer Kombination fast mit jeder Zielgruppe möglich ist.

\section{FRAGEN ZUR GIF}

\subsection{Die Beziehungen zu den Untersektoren}

Es besteht bei den Entwicklungsorganisationen ein Konsens darüber, dass bei der GIF nur kleinste und kleine bis mittlere Unternehmen gefördert werden sollen, d.h., dass eine Abgrenzung nach oben zu den grösseren und grossen Betrieben notwendig ist, auch wenn bei dieser Abgrenzung im Einzelnen unterschiedliche Kriterien angewendet werden. Dahinter steht mindestens eine von drei Annahmen: einmal, dass grössere Betriebseinheiten sich selber helfen können und keine Unterstützung brauchen; dann, dass solche Einheiten schon genügend Förderung aus andern Bereichen erhalten und von der EZA nicht bedacht werden müssen; und schliesslich, dass grössere und Grossbetriebe aus verschiedenen Gründen der wirtschaftlichen Struktur eines Entwicklungslandes nicht angepasst sein mögen. Jedenfalls schafft die GIF innerhalb der Wirtschaft grundsätzlich zwei Untersektoren: einen förderungsbedürftigen und einen nicht unterstützungswürdigen.

Durch diese Abgrenzung der beiden Untersektoren wird die zwischen ihnen bestehende Interdependenz vernachlässigt. Ein Eingehen auf diese Interdependenz würde jedoch die Frage nach ihrem jeweiligen Verhältnis zueinander erlauben: Ist dieses komplementär oder stehen die beiden Untersektoren in einem Konkurrenzverhältnis zueinander? Können Synergieeffekte erwartet werden?

Sicher ist, dass der moderne, kapitalintensive und von aussen kontrollierte Untersektor durch Privatinteressen aus den Industriestaaten (Privatinvestitionen, Know-how Transfer, weitere Geschäftsbeziehungen) volumenmässig mehr gefördert wird, als der handwerklich-kleinindustrielle Unter- 
sektor mit den beschränkten Mitteln, die der EZA zur Verfügung stehen. Welche Relevanz hat die GIF der EZA gemessen an den Bedürfnissen an Arbeitsplätzen, die im nicht-landwirtschaftlichen Bereich bei hohen Zuwachsraten der Gesamt- und besonders der Stadtbevölkerung geschaffen werden müssen? Eine signifikante Veränderung der Struktur des gesamten Sektors wird nur dann möglich sein, wenn die Mittel, welche diesem Sektor von der EZA zufliessen, massiv erhöht werden und/oder eine kräftige Verlagerung der privatwirtschaftlichen Kapitalflüsse zugunsten des kleinbetrieblichen Sektors stattfindet. Damit diese Verlagerung möglich wird, müsste allerdings die Attraktivität dieses Untersektors auf mirakulöse Art für private Investoren erhöht werden.

\subsection{Die Dominanz betriebswirtschaftlicher Konzepte}

Wie wenn es selbstverständlich wäre, wird die Diskussion über die GIF von einer betriebswirtschaftlichen Optik dominiert. Dahinter steht die Ueberlegung, dass die Erhaltung und Förderung kleinerer Unternehmen, die sich in einem Markt zu behaupten wissen, auch marktmässiges Verhalten mit entsprechenden Konsequenzen auf der betrieblichen Ebene verlangt : ein günstiges Aufwand/Ertrags-Verhältnis, eine bestimmte Arbeitsproduktivität, ein gewisses Umsatzvolumen, die Kenntnis und Durchführung von Kostenkalkulationen. Letzlich geht es dabei um eine Optimierungsfrage, um Gewinnmaximierung in einem weiteren Sinne. Darob werden komplexe, entwicklungspolitische Zielsetzungen oft aus dem Auge verloren.

Das Vorherrschen von betriebswirtschaftlichen Ueberlegungen erstaunt insofern, als in der übrigen EZA die Notwendigkeit eines vieldimensionalen Verständnisses von Entwicklungsfragen immer stärker anerkannt wird: die Beachtung sozialer, kultureller, ökologischer und anderer Zusammenhänge. Bestimmen nicht ethnische, soziale, familiäre Gesichtspunkte oft die Beziehungen zwischen den Mitarbeitern innerhalb eines Kleinbetriebes und zwischen den Kleinbetrieben unter sich? Wird das Wirtschaftsverhalten nicht durch solche Faktoren wesentlich geprägt?

Viele der betriebswirtschaftlichen Merkmale eines Unternehmens erscheinen handlich und objektiv, weil sie gut definierte und messbare Grössen darstellen. Aber zeichnet sich nicht gerade der Bereich der kleinsten und kleinern Unternehmen durch lückenhafte oder fehlende Betriebsdaten aus: fehlende Buchhaltungen, mangelhafte Statistiken, Verdeckung von wesentlichen Informationen?

Eine ganz besondere Bedeutung kommt bei der GIF den institutionellen Rahmenbedingungen zu; dies gilt insbesondere bei der Gestaltung der monetären Politik eines Landes, bei der Festsetzung von Kredithöhen und Zinssätzen und bei der administrativen Abwicklung dieser Dienstleistungen. Werden institutionelle Fragen genügend beachtet? Solche Fragen sind: Was tun die öffentlichen und privaten Institutionen im Umfeld der von GIFMassnahmen anvisierten Untersektoren? Welche Institutionen fördern, welche bremsen die Entwicklung möglicher Zielgruppen? Sind Hilfsfunktionen 
zur GIF, wie Information/Dokumentation, Beratung, Forschung, Rechtssetzung und Policy-Definition institutionell verankert?

\subsection{Das Verhältnis zur sozialen Frage innerhalb des Sektors}

Wie verhält sich die GIF zu auftauchenden sozialen Problemen innerhalb des Sektors? Die Frage stellt sich weniger dort, wo es um das "Self-employment» Einzelner geht. Sie stellt sich aber in den Unternehmen, und seien sie noch so klein, wo für einen Unternehmer Lohnarbeit geleistet wird und wo es folglich um das Aushandeln von Vertragsbedingungen zwischen unterschiedlichen Interessen geht. Mit GIF-Massnahmen wird oft das Ziel verfolgt, geringe Einkommen zu erhöhen, eine egalitärere Einkommensverteilung zu suchen, die Arbeitsplatzsicherung zu verbessern. Solche Ziele dekken sich mit den klassischen Gewerkschaftszielen. Geht man davon aus, dass Gewerkschaften Wesentliches zur Erreichung dieser Ziele beitragen können und schon beigetragen haben, dann erstaunt die Ausklammerung der Gewerkschaftsfrage und der Frage nach der sozialen Verantwortung der Einzelunternehmer in der GIF-Diskussion. Kaum ist im Rahmen der GIF von besonderen Massnahmen, welche nicht nur den Kleinunternehmer, sondern auch seine Arbeiter und Lehrlinge über das technisch/kaufmännische hinaus qualifizieren, die Rede ; kaum werden explizit gewerkschaftliche Organisationen in diesem Sektor unterstützt. Diese Unterstützung wird, von der hier zur Diskussion stehenden Förderung abgekoppelt, nur von kirchlichen und politischen Hilfsorganisationen geleistet.

Stellt man sich solche Fragen bei der Konzeption der GIF nicht, läuft man Gefahr, langfristig Arbeitsplätze um «jeden Preis» zu schaffen und zu erhalten, auch wenn damit schlechte Lohn- und Arbeitsbedingungen verknüpft sind. Es würden somit Unternehmen gefördert und am Leben erhalten werden, bei denen nationale und internationale Arbeitsrechte und Standards nicht gelten. Muss deshalb die GIF zugunsten von kleinen und mittleren Unternehmen nicht gleichzeitig mit einer Komponente zur Gewerkschaftsförderung erweitert werden?

\subsection{Die Partizipation der Betroffenen}

Wie gross ist die Partizipation der Zielgruppen bei GIF-Massnahmen? Es besteht ein breiter Konsens darüber, dass die Bedürfnisse einer Zielgruppe dann am besten erkannt und befriedigt werden können, wenn diese Gruppen entscheidend Entwicklungsmassnahmen mittragen, d.h., sie kennen, mitdefinieren, akzeptieren und mitgestalten. Je diffuser, marginaler, unorganisierter eine Gruppe, desto schwieriger ist es, sie an einem Entwicklungsprozess zu beteiligen und desto langsamer kommt diese Beteiligung zustande; das ist mit ein Grund dafür, weshalb in der EZA etwa auf besser zugängliche Zielgruppen ausgewichen wird, wo sich Erfolge schon mittelfristig ausweisen lassen. Ist dies eine Erklärung dafür, dass auch in diesem 
Sektor die Frauen als spezifische Gruppe anscheinend kaum an Förderungsmassnahmen partizipieren, obwohl sie an den traditionellen handwerklichen Tätigkeiten (z.B. Textilfärben) und im Kleinhandel einen erheblichen Anteil haben?

Daneben gibt es eine zweite Frage in diesem Zusammenhang: Wie müssen Projektziele und der Projektdesign zugunsten des "Micro-Bereiches» aussehen, damit die Partizipation auch während des Projektablaufs gewährleistet ist? Die Erwerbsformen zeichnen sich in diesem Bereich durch vielfältige Kombinationen und Improvisation auf tiefster Stufe aus. Wie sieht ein Projekt aus, das diese Qualitäten aufnimmt und verwertet? Die Gefahr besteht, dass unsere Vorstellungen von Kontinuität, Ordentlichkeit und Voraussehbarbeit die Ueberlebensformen in der Marginalität schwächen, statt sie zu verbessern und dass die "Begünstigten» vollkommen von Wertvorstellungen und Verhaltenserwartungen abhängig gemacht werden, die von aussen an sie herangetragen worden sind.

\subsection{Die Wahl der anzuwendenden Technik}

Die Förderungsmassnahmen betreffen sowohl Ausbildung und Können der Produzenten als auch ihre Produktionsmittel, insbesondere ihre Werkzeuge und Maschinen. Die Einführung technischer Veränderungen zielt auf eine Erhöhung der Produktivität, eine Verbesserung der Qualität, oft auch auf eine Ersparnis bei den Ressourcen ab. Diese Veränderungen können jedoch nur wirksam werden, wenn sie in einem Lernprozess vom Produzenten angeeignet und angewendet werden. Dabei stellen sich die Fragen nach der Angepasstheit im Herstellungsprozess durch verschiedene Produzenten und Institutionen. Die Frage nach der Angepassheit kann nur kontextabhängig beantwortet werden und weit mehr als die eigentliche technologische Lösung entscheidet der sie begleitende Lernprozess ihre erfolgreiche Anwendbarkeit.

Damit stellen sich für die im Rahmen der GIF angewandte Technik drei Fragen: Wie können in den Industrieländern entwickeltes Know-how und Produktionsmittel kontextgerecht für die Verwendung in den Entwicklungsländern adaptiert werden? Wie können allenfalls bei uns verloren gegangenes Wissen und die entsprechenden Produktionsmittel wieder angewandt werden? und schliesslich: Wie können lokal vorhandenes Wissen und die entsprechenden Produktionssmittel für eine neue, kontextgerechte Technik nutzbar gemacht werden?

\subsection{Der Einfluss der Marktverhältnisse}

Alle Massnahmen der GIF orientieren sich an einer innersektoriellen Stärkung des Handwerks und der Kleinindustrie. Diese Förderung stösst aber rasch an ihre Grenzen, wenn die wirtschaftlichen Aktivitäten des Sektors marktabhängig sind und seine Stabilisierung und Strukturierung eine Nach- 
fragesituation verlangt, die selbst möglichst geringen konjunkturellen Schwankungen unterliegt und ein Wachstumspotential aufweist. Das Potential lässt sich dann nutzen, weil die gewerblich/industrielle und die private Nachfrage erhöht werden kann. Dies setzt vermehrte wirtschaftliche Aktivitäten, eine gesteigerte Kaufkraft und einen auf die Produktionsmöglichkeiten des Sektors orientierten Konsum voraus.

In der gegenwärtigen Phase geringen wirtschaftlichen Wachstums des internationalen Handels und der grassierenden Zahlungsbilanzprobleme vieler ärmerer Entwicklungsländer mag die regionale und nationale Marktkonjunktur der GIF entgegenkommen. Die liberalen internationalen wirtschaftspolitischen Rahmenbedingungen und die in manchem Entwicklungsland angewandten Anpassungsmassnahmen lassen jedoch ein optimistisches Szenario hinsichtlich des Potentials und der Dynamik der GIF als allzu vermessen erscheinen und setzen ihr ihre eigenen Grenzen.

\section{LITERATURHINWEISE}

Botschaft des Bundesrates über die Weiterführung der technischen Zusammenarbeit und der Finanzhilfe zugunsten von Entwicklungsländern, Bern 1984.

Botschaft des Bundesrates über die Weiterführung der Finanzierung von wirtschaftsund handelspolitischen Massnahmen im Rahmen der Entwicklungszusammenarbeit, Bern 1986.

Canadian International Development Agency, Industrial Series: Enterprise Development in Developing Countries, Canadian Perspectives (Vol. 1), 1986.

Direktion für Entwicklungszusammenarbeit und humanitäre Hilfe (DEH), 10-Jahresbericht (Entwurf), 1986.

FUNDES-Dokumentation, Niederurnen, 1986.

ILO, The Promotion of Small and Medium-Sized Enterprises (International Labour Conference, 72. session), Geneva 1986.

IUED, Genève-Afrique 1984/1: Les artisans urbains en Afrique, Genf.

IUED, Cahiers de I'IUED: Demain l'artisanat, Genf 1986.

Lachaud J.-P., Le développement spontané, Paris 1985.

Page, J.M.jr/Steel, W.F.: Small Enterprise Development - Economic Issues from African Experience, World Bank Technical Paper Nr. 26, Washington, 1984.

Revue Canadienne d'études du développement, Vol. VI, No 1, 1985: Le travail informel urbain: Une évaluation de la littérature, Université d'Ottawa, Ottawa.

Schneider-Barthold W., Entwicklung und Förderung des Kleingewerbes in der Dritten Welt, München 1984.

Swissaid: Guinea-Bissau, Rapports annuels 1981-1985, Bern.

Swisscontact: Jahresbericht Costa-Rica 1981-1985, Zürich.

Swisscontact: Gewerbeförderung - Richtlinien für Projekte, Zürich.

UNIDO : Investment-Promotion-Service, Dokumentation, Zürich. 